\title{
Chronic mTOR activation induces a degradative smooth muscle cell phenotype
}

\author{
Guangxin Li, ${ }^{1,2}$ Mo Wang, ${ }^{1}$ Alexander W. Caulk, ${ }^{3}$ Nicholas A. Cilfone, ${ }^{4}$ Sharvari Gujja, ${ }^{4}$ Lingfeng Qin, ${ }^{1}$ Pei-Yu Chen, ${ }^{5}$ \\ Zehua Chen, ${ }^{4}$ Sameh Yousef, ${ }^{1}$ Yang Jiao, ${ }^{1}$ Changshun He, ${ }^{1}$ Bo Jiang, ${ }^{1}$ Arina Korneva, ${ }^{3}$ Matthew R. Bersi, ${ }^{3}$ Guilin Wang, ${ }^{6}$ \\ Xinran Liu, ${ }^{7,8}$ Sameet Mehta, ${ }^{9}$ Arnar Geirsson, ${ }^{1,10}$ Jeffrey R. Gulcher, ${ }^{4}$ Thomas W. Chittenden, ${ }^{4}$ Michael Simons, ${ }^{5,10}$ \\ Jay D. Humphrey, ${ }^{3,10}$ and George Tellides ${ }^{1,10,11}$
}

'Department of Surgery, Yale School of Medicine, New Haven, Connecticut, USA. ²Department of Breast and Thyroid Surgery, Peking University Shenzhen Hospital, Shenzhen, Guangdong Province, China. ${ }^{3}$ Department of Biomedical Engineering, Yale School of Engineering and Applied Science, New Haven, Connecticut, USA. ${ }^{4}$ Computational Statistics and Bioinformatics Group, Advanced Artificial Intelligence Research Laboratory, WuXi NextCODE, Cambridge, Massachusetts, USA. ${ }^{5}$ Internal Medicine, ${ }^{6}$ Molecular Biophysics and Biochemistry, and ${ }^{7}$ Cell Biology, Yale School of Medicine, New Haven, Connecticut, USA ${ }^{8}$ Center for Cellular and Molecular Imaging, EM Core Facility, Yale School of Medicine, New Haven, Connecticut, USA. ${ }^{9}$ Cenetics and ${ }^{10 P r o g r a m ~ i n ~ V a s c u l a r ~ B i o l o g y ~ a n d ~ T h e r a p e u t i c s, ~ Y a l e ~ S c h o o l ~ o f ~ M e d i c i n e, ~}$ New Haven, Connecticut, USA. "Veterans Affairs Connecticut Healthcare System, West Haven, Connecticut, USA.

Smooth muscle cell (SMC) proliferation has been thought to limit the progression of thoracic aortic aneurysm and dissection (TAAD) because loss of medial cells associates with advanced disease. We investigated effects of SMC proliferation in the aortic media by conditional disruption of Tsc1, which hyperactivates mTOR complex 1. Consequent SMC hyperplasia led to progressive medial degeneration and TAAD. In addition to diminished contractile and synthetic functions, fate-mapped SMCs displayed increased proteolysis, endocytosis, phagocytosis, and lysosomal clearance of extracellular matrix and apoptotic cells. SMCs acquired a limited repertoire of macrophage markers and functions via biogenesis of degradative organelles through an mTOR/ $\beta$-catenin/MITF-dependent pathway, but were distinguishable from conventional macrophages by an absence of hematopoietic lineage markers and certain immune effectors even in the context of hyperlipidemia. Similar mTOR activation and induction of a degradative SMC phenotype in a model of mild TAAD due to Fbn1 mutation greatly worsened disease with near-uniform lethality. The finding of increased lysosomal markers in medial SMCs from clinical TAAD specimens with hyperplasia and matrix degradation further supports the concept that proliferation of degradative SMCs within the media causes aortic disease, thus identifying mTOR-dependent phenotypic modulation as a therapeutic target for combating TAAD.

\section{Introduction}

Thoracic aortic aneurysm and dissection (TAAD) afflict both sexes, young and old alike, and can be lethal due to rupture. These conditions are attributed to cellular and extracellular matrix (ECM) abnormalities of the medial layer of the aortic wall (1). This layer contains numerous alternating concentric elastic lamellae and smooth muscle cells (SMCs) that are embedded within ECM constituents, including collagens, fibrillins, and glycosaminoglycans. Although SMC death is frequent in end-stage pathology (2), it may not necessarily initiate disease. Indeed, we found that the number of SMCs increases in clinical specimens of sporadic TAAD, particularly in small lesions (3). Although these descriptive observations cannot infer disease causality, we hypothesized that SMC proliferation could either be an adaptive response to increased hemodynamically induced stress within a dilated aorta or exacerbate

\section{Related Commentary: p. 1096}

Conflict of interest: The authors have declared that no conflict of interest exists. Copyright: ( 2020, American Society for Clinical Investigation.

Submitted: June 13, 2019; Accepted: December 3, 2019; Published: February 10, 2020. Reference information: / Clin Invest. 2020;130(3):1233-1251.

https://doi.org/10.1172/JCl131048. disease progression depending on cell phenotype. Unless new, phenotypically similar SMCs replace dying cells precisely, additional progeny will require accommodation within the concentric elastic lamellae that do not increase in number. If daughter cells align side-to-side, they may disrupt homeostatic interactions of SMCs with the ECM; if instead they align end-to-end, this will compel a circumferential growth of the media that could contribute to dilatation. These simple considerations challenge the premise that SMC proliferation represents a therapeutic strategy for TAAD (4, 5). Importantly, the question of phenotype of the proliferating cells is central to disease outcome and demands further investigation.

Elucidation of genetic etiologies for TAAD has reinforced a possible pathological role for SMC proliferation associated with an impairment of the contractile phenotype. For example, mutations of ACTA2, which encodes smooth muscle $\alpha$-actin (SMA), result in TAAD characterized by focal areas of proliferating SMCs in the aortic media and marked medial hyperplasia in small arteries (6). Similarly, mutations of $M Y H 11$, which encodes smooth muscle myosin heavy chain (SMMHC), lead to TAAD associated with focal losses but also focal hyperplasia of medial SMCs (7). Further support for a concept of detrimental hyperplasia is the rare occurrence of ruptured aortic aneurysms in young patients with tuberous sclerosis $(8,9)$. This multisystem genetic 
disease is caused by mutations in TSC1 or TSC2, which encode the proteins hamartin and tuberin, respectively, that together form the tuberous sclerosis complex that acts as a tumor growth suppressor by inhibiting mTOR, a key kinase regulating cellular growth and differentiation (10). Among diverse manifestations, individuals with tuberous sclerosis present with mesenchymal tumors consisting of proliferating SMCs and characterized by mTOR hyperactivation. A second-hit somatic mutation to the unaffected allele is postulated for tuberous sclerosis lesions, an event that may occur with insufficient frequency in vascular SMCs to induce overt aortic disease. Nevertheless, histological analysis of a rare thoracoabdominal aneurysm in a child with a TSC2 mutation revealed SMC hyperplasia in the inner media with diminished SMA expression and extensive fragmentation of elastic fibers (11). In accompanying studies on $T s c 2^{+/-}$mice, cultured SMCs also showed increased proliferation and decreased contractile protein expression with an exaggerated neointimal formation following wire injury. Although the hemizygous phenotype is mild, these findings support the concept that mTOR drives SMC dedifferentiation, consistent with in vitro studies that the mTOR inhibitor rapamycin promotes SMC differentiation toward a contractile phenotype (12).

Vascular SMCs demonstrate marked phenotypic modulation, originally observed to range from contractile in quiescent mature arteries to proliferative and synthetic in disease or injury. Dedifferentiation can thus involve loss of contractile molecules and increased synthesis of ECM (13). Phenotypic changes in SMCs are not constrained along this contractile-to-synthetic spectrum, however. Chondrogenic and osteogenic phenotypes lead to accumulated glycosaminoglycans and calcification, while transdifferentiation into other cell types is also possible. Macrophage-like cells derived from SMCs accrue in atherosclerotic lesions and contribute to neointima expansion; they are not detected in the media, however (14). Previous studies of medial SMC changes in TAAD have described loss of contractile proteins and acquisition of synthetic properties, as described for intimal SMCs in atherosclerotic disease (15-17).

In this study, we investigated whether SMC proliferation promotes or prevents TAAD and how MTOR modulates SMC phenotype. Toward this end, we used postnatal SMC-specific disruption of $T s c 1$ in mice as well as treatment with the mTOR inhibitor rapamycin. The data reveal that an mTOR-dependent proliferation of SMCs rich in lysosomes and expressing a subset of macrophage markers causes TAAD. Moreover, hyperactivation of mTOR exacerbates disease in a prior mouse model of mild TAAD. There is, therefore, strong motivation to target the mTOR-dependent degradative SMC phenotype when seeking to treat diverse thoracic aortopathies.

\section{Results}

Postnatal disruption of Tsc1 in SMCs causes progressive aortic disease. To investigate effects of increased mTOR activity and SMC proliferation in the aortic wall, we bred mice with 3 transgenes: (a) the mTORC1 inhibitor Tsc1, with exons 17 and 18 flanked by loxP sites (18); (b) Cre recombinase fused with a modified estrogen receptor under control of a smooth muscle-specific Myh11 promoter (19); and (c) the $m T / m G$ double-fluorescent reporter that globally expresses membrane-targeted tdTomato, a variant red fluorescent protein (RFP), except where excised by Cre to express membrane-targeted GFP (20). DNA recombination was induced by tamoxifen treatment of 1.5-week-old Tsc $1^{f l / f l} M y h 11-C r e E R^{T 2} m T / m G$ mice, while control litters were treated with corn oil vehicle. Efficiency and specificity of recombination were confirmed by disruption of Tsc1, loss of its product hamartin, selective expression of GFP instead of RFP by medial cells, and increased mTOR activity in all regions of the aorta (Supplemental Figure 1; supplemental material available online with this article; https://oi.org/10.1172/ JCI131048DS1). In certain experiments, control SMCs expressing GFP were obtained from tamoxifen-treated Myh11-CreER ${ }^{T 2}$ $m T / m G$ mice lacking $T s c 1^{f l / f l}$.

Animals with conditional disruption of $T s c 1$ were serially sacrificed and their aortas compared with controls. At 12 weeks of age, $20 \%$ of thoracic aortas exhibited aneurysms (greater than 1.5-fold fusiform dilatation or any saccular dilatation) and dissections (blood within the vessel wall) (Figure 1, A and B). Although the unloaded ascending aortas were dilated with increased mass, they were not elongated (Figure 1C). There were no differences in descending thoracic aorta dimensions or cardiac and body mass (Supplemental Figure 2A), and vessels other than the thoracic aorta had a normal appearance (not shown). Disease manifestations in the thoracic aorta progressively worsened. The incidence of TAAD increased to $33 \%$ at 24 weeks and $55 \%$ by 36 weeks, at which time ascending segments were also elongated and descending segments dilated (Figure 1D and Supplemental Figure 2, B and C). Mean blood pressure and pulse pressure, but not heart rate, were significantly lower, thus excluding hypertension as a cause of the aortic pathology (Figure 1E). Antemortem ultrasound examination verified larger-diameter ascending aortas with diminished distension during the cardiac cycle (Figure 1, F and G and Supplemental Figure 2D). Excised ascending segments demonstrated blunted responses to vasoconstrictors that worsened considerably with duration of $T s c 1$ disruption, translating to impaired reduction of pressure-induced circumferential stress on the vessel wall from decreased vasoconstriction (Figure $1 \mathrm{H}$ and Supplemental Figure 2, $\mathrm{E}$ and $\mathrm{F}$ ). Biomechanical analysis also revealed progressive losses of elastically stored energy, a key functional metric of the aorta, and late increases in circumferential material stiffness (Supplemental Figure 2, G and $\mathrm{H}$ and Supplemental Tables 1 and 2), which often associates with aneurysms (21). This progressive vascular dysfunction ex vivo suggested intrinsic vessel wall defects.

SMC proliferation and elastin fragmentation characterize the aortic pathology. Vessel sections were analyzed by histomorphometry to determine cellular and ECM changes. Ascending aortas with conditional Tsc1 disruption exhibited luminal dilatation and medial expansion at 12 weeks (Figure 2, A and B); a larger circumference and thicker wall contributed to the increased medial area (Supplemental Figure 3A). The media also had more cells per cross section, without any change in cell density (Figure 2, C and D). SMC proliferation was confirmed by increased BrdU uptake at 5 and 12 weeks, whereas SMC apoptosis, as assessed by a TUNEL assay, was initially low and not increased until 24 weeks (Figure 2, E-H and Supplemental Figure 3B). Strikingly, there was increased elastin fragmentation in all ascending segments and partial elastin loss throughout the thoracic aorta at 12 weeks (Figure 2, I and J), 
A

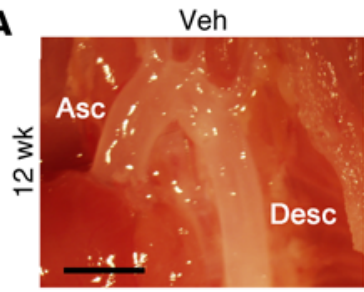

Tmx

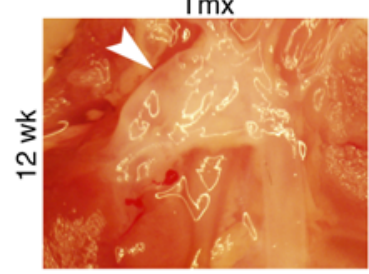

B
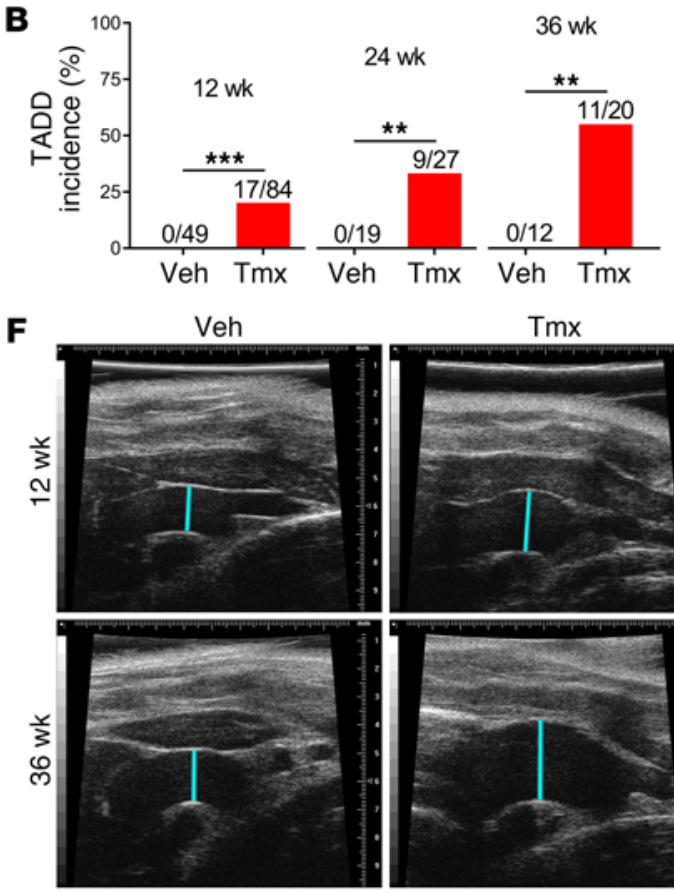
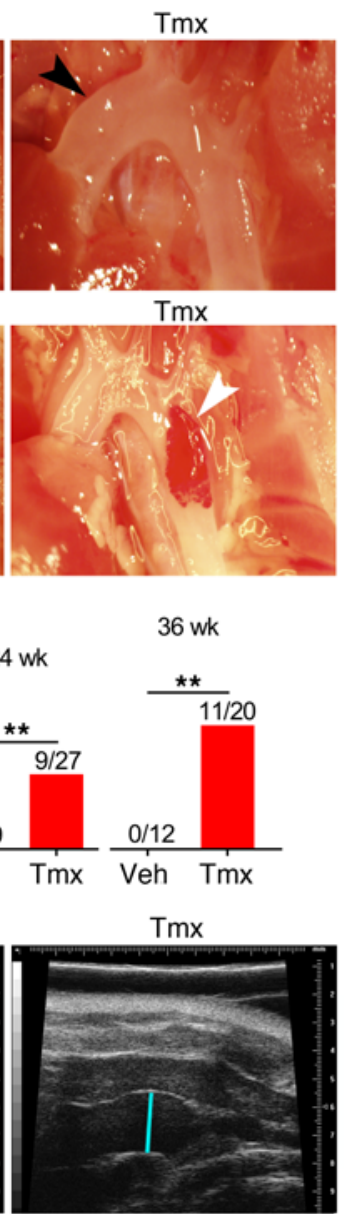

$\operatorname{Tmx}$

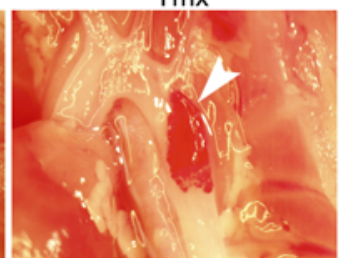

6 wk

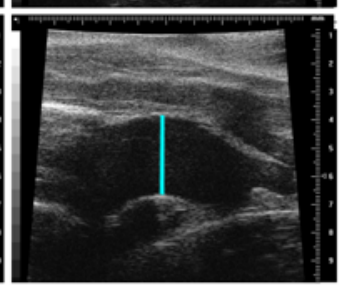

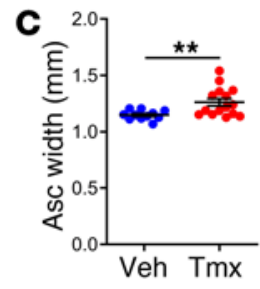

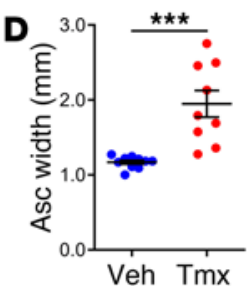

E

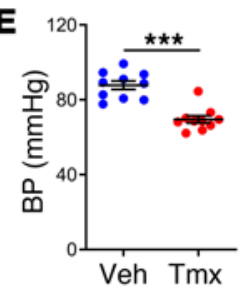

G
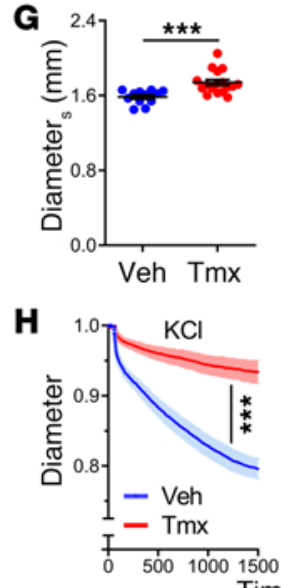
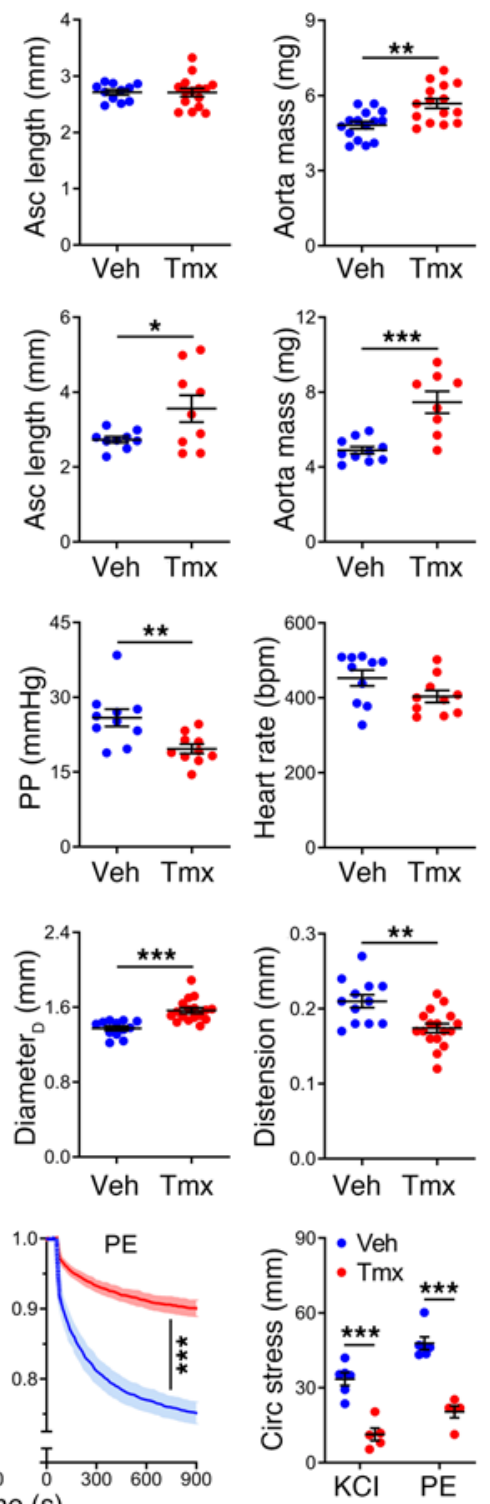

Figure 1. Tsc1 deletion in SMCs results in progressive aortic disease and dysfunction. TsC ${ }^{f / / f l} M y h 11-C r e E R^{T 2} \mathrm{mT} / \mathrm{mG}$ mice were treated with tamoxifen $(\operatorname{Tmx})$ or vehicle (Veh) at 1.5 weeks of age and their thoracic aortas were serially examined. (A) In situ examination of ascending (Asc) and descending (Desc) thoracic aortas showing frequent mild dilatation (black arrow) with occasional aneurysms or dissections (white arrows) at 12 weeks. Scale bar: 2 $\mathrm{mm}$. (B) Incidence of TAAD at 12, 24, and 36 weeks. (C) Width and length of unpressurized ascending segments and mass of thoracic aortas at 12 weeks $(n=11-15)$. (D) Aortic dimensions at 36 weeks $(n=8-10)$. (E) Mean blood pressure (BP), pulse pressure (PP), and heart rate at 12 weeks $(n=10)$. (F) Ultrasound examination showing ascending aorta diameter (blue lines) at 12 and 36 weeks. (G) In vivo ascending aorta diameter at end-systole (Diameter ${ }_{\mathrm{S}}$ ), end-diastole (Diameter ${ }_{D}$ ), and distension at 12 weeks $(n=12-17)$. (H) Ascending aorta outer diameter, normalized to pretreatment value, measured ex vivo in response to $100 \mathrm{mM} \mathrm{KCl}$ or $1 \mu \mathrm{M}$ phenylephrine (PE) and the associated percentage reduction in circumferential (Circ) stress at 12 weeks $(n=5-6)$. Data are represented as individual values with mean \pm SEM bars or as line plots with SEM. ${ }^{*} P<0.05,{ }^{* *} P<0.01,{ }^{* * *} P<0.001$ for Tmx vs. Veh by Fisher's exact test (B), $t$ test (C-E and $\mathbf{C}), 2$-way repeated-measures ANOVA (H, left and middle), or 2-way ANOVA (H, right).

consistent with the aforementioned decreased elastic energy storage. Elastin breaks were greater in the subset of vessels with TAAD and vessel wall architecture was more disordered at 36 weeks when most thoracic aortas were aneurysmal or dissected (Supplemental Figure 3C). Elastin degradation was minimal in arteries other than the thoracic aorta, and markedly less in the ascending aorta if induction of Cre activity was delayed to 12 weeks of age; degradation was not a result of tamoxifen administration or GFP expression, as it did not occur in mutants lacking Myh11-CreER ${ }^{T 2}$ or $T s c 1^{f l / f l}$ (Supplemental Figure 3, D-G). Collagen did not accumu- late within the media, except in focal areas with severe disruption of elastin (Supplemental Figure 3, H and I). Electron microscopy at 24 weeks confirmed elastic fiber attenuation, while SMCs displayed disordered cytoplasmic filaments and more perinuclear organelles that extended peripherally (Figure 2K). In summary, cellular and ECM abnormalities contributed to the observed medial expansion and degeneration.

Loss of Tsc1 causes mTORC1 hyperactivation, whereas mTOR inhibition rescues the aortic phenotype. Perturbations in $\mathrm{mTOR}$ signaling were assessed by Western blotting of thoracic aorta lysates after 
A

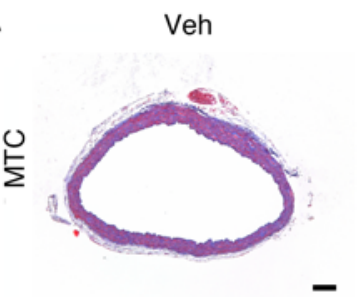

C

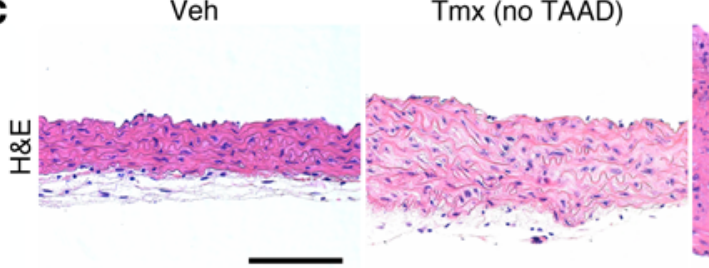

E

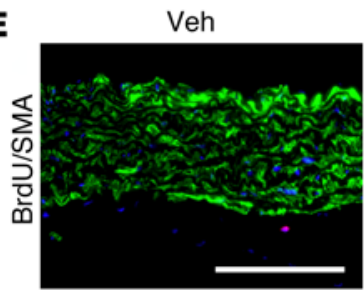

I

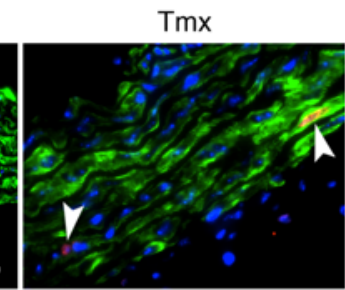

Tmx (no TAAD)
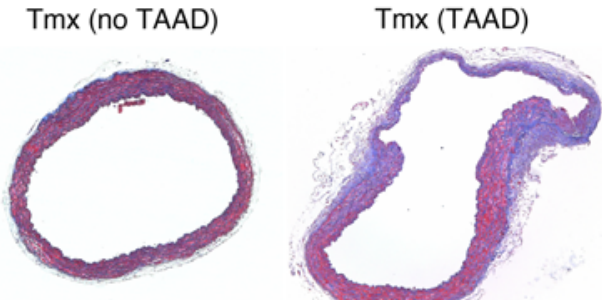

$\operatorname{Tmx}(T A A D)$
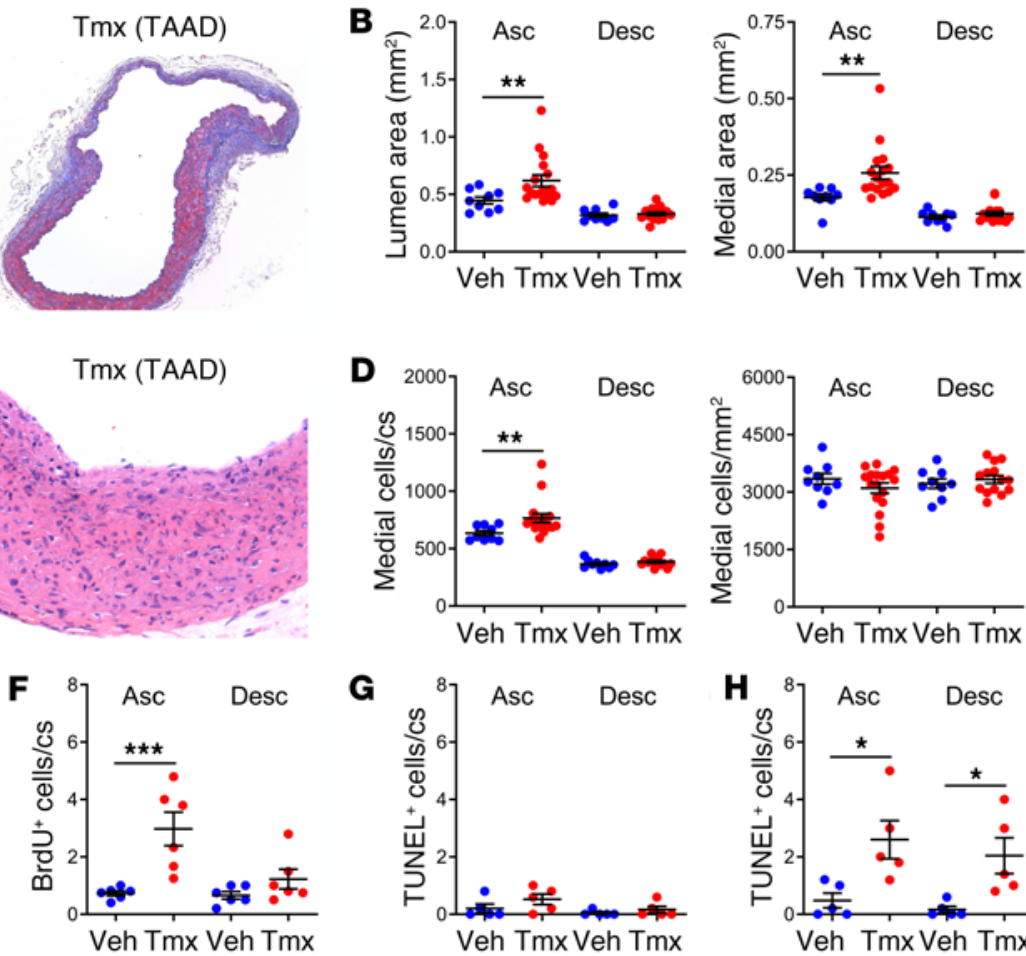

G
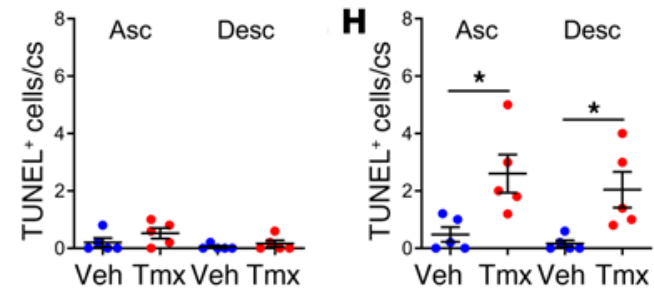

Tmx (TAAD)

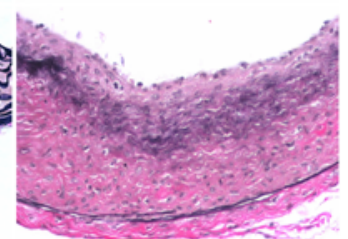

J
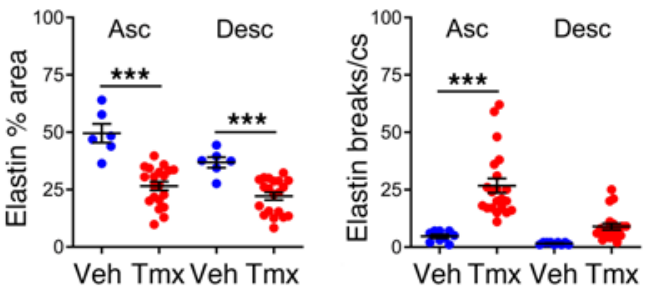

$\mathbf{K}$
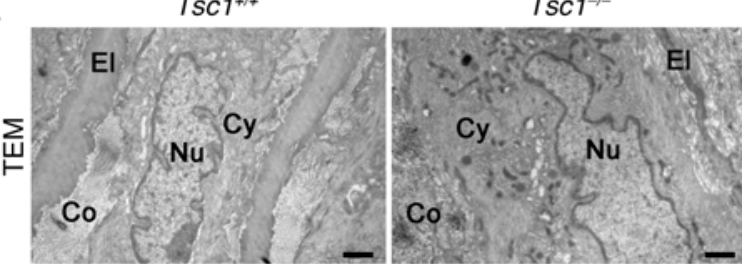

$\operatorname{TSC1}^{+/+}$
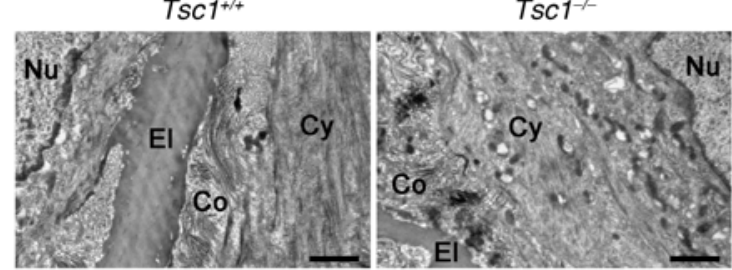

Figure 2. Aortic pathology is characterized by SMC proliferation and elastin fragmentation. TSC1 $1^{f l / f l} M y h 11-C r e E R^{T 2} m T / m G$ mice were treated with tamoxifen (Tmx) or vehicle (Veh) at 1.5 weeks of age and their ascending (Asc) and proximal descending (Desc) thoracic aortas were examined by histology at 12 weeks of age; representative photomicrographs of ascending aortas without or with TAAD are shown. Scale bars: $100 \mu$ m. (A) Masson's trichrome (MTC) stains and (B) lumen and media area $(n=9-17)$. (C) H\&E stains and (D) number of medial cells per cross section (cs) or area ( $n=9-17)$. (E) BrdU reactivity (red color marked by arrows), SMA expression (green color), and DAPI-labeled nuclei (blue color) in subset of mice receiving BrdU for 2 weeks and $(\mathbf{F})$ number of BrdU ${ }^{+}$medial cells per cross section $(n=6)$. Number of TUNEL ${ }^{+}$medial cells per cross section at $(\mathbf{G}) 12$ weeks and $(\mathbf{H}) 24$ weeks $(n=5)$. (I) Verhoeff-Van Gieson (VVG) stains and (J) elastin fraction of media area and number of elastin breaks per cross section at 12 weeks ( $n=6-21)$. Data are represented as individual values with mean \pm SEM bars. ${ }^{*} P<0.05,{ }^{*} P<0.01,{ }^{* *} P<0.001$ for Tmx vs. Veh by 2 -way ANOVA. (K) Transmission electron

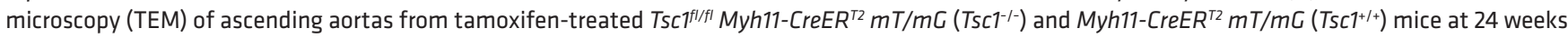
showing elastin attenuation, loss of cytoplasmic filaments, and more cytoplasmic organelles extending from perinuclear region to periphery. El, elastin; Co, collagen; Nu, nucleus; Cy, cytoplasm. Scale bars: $1 \mu \mathrm{m}$.

overnight fasting of animals. Disruption of Tsc1 increased activation of the mTOR complex 1 (mTORC1) at 3 weeks of age, as indicated by greater phosphorylation of its effectors, S6K, S6, and 4EBP1 (Figure 3A). Phosphorylation of the mTORC2 substrate Akt at serine 473 and expression of contractile proteins, such as SMMHC, SMA, and SM22, were not significantly altered at this early time, although there was less activation of a contractile regulator, myosin light chain 2 (MLC2) (Supplemental Figure 4A). Akt phosphorylation diminished by 12 weeks, likely via feedback inhibition by S6K as previously reported (22), and expression of contractile molecules also diminished (Figure 3B), consistent with the functional finding of impaired contractility. Altered mTOR signaling did not depend on the hemodynamic environment because similar findings persisted in vitro, there was no downregulation of mTORC1 signaling 
A
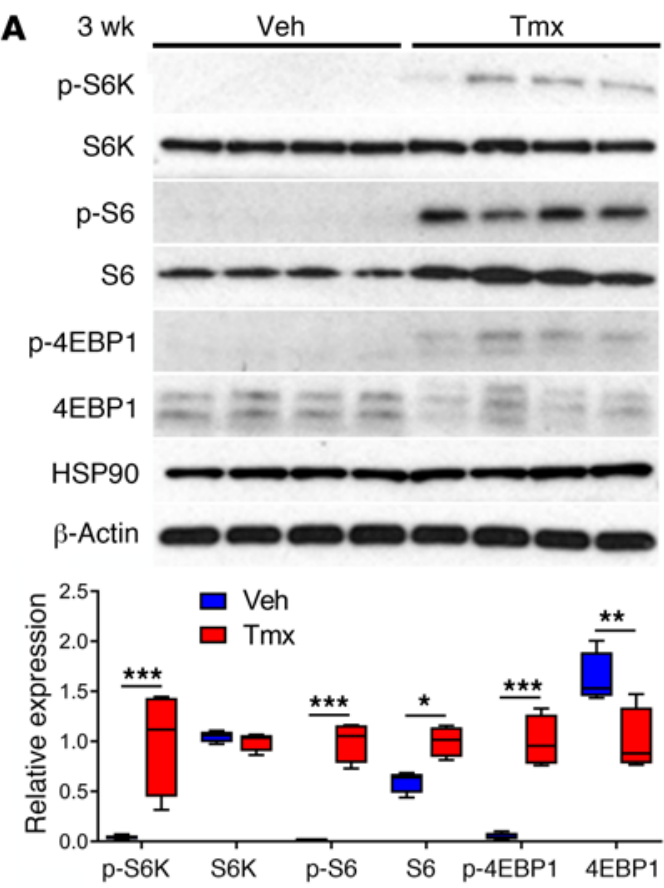

C

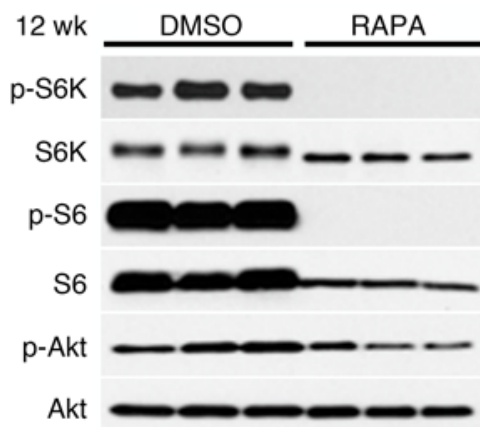

SMMHC $\backsim U=\boldsymbol{U}=$
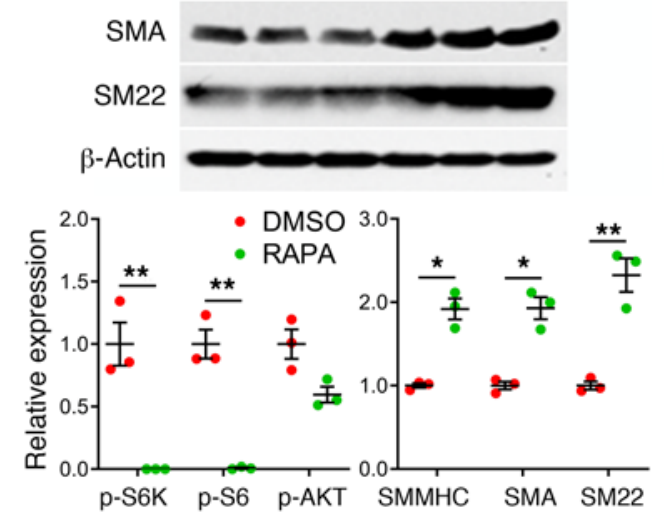

B
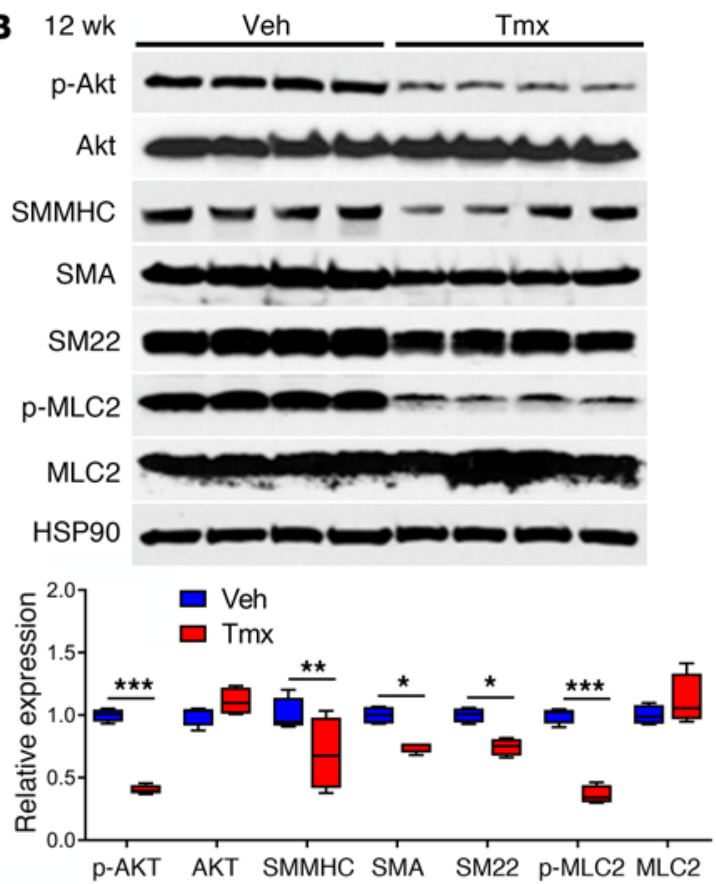

D
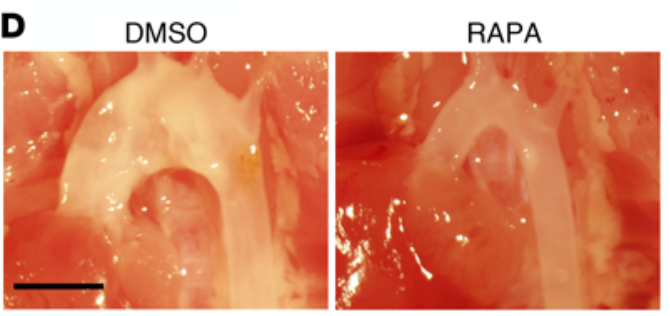

E

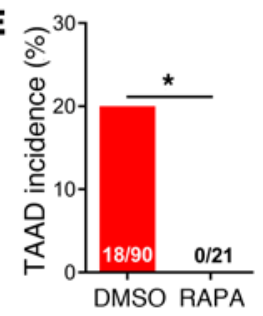

F

DMSO

RAPA
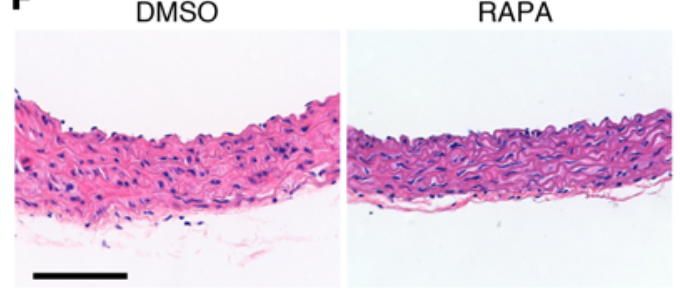

G

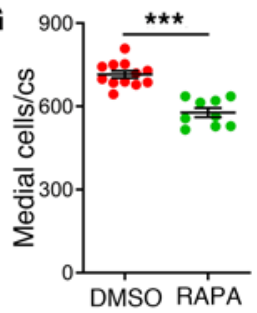

H

DMSO

RAPA
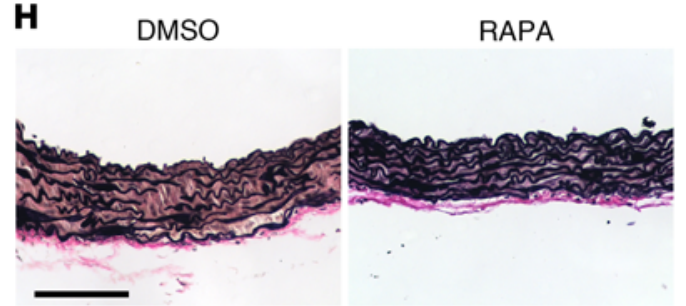

I

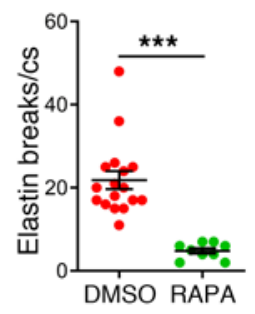

Figure 3. mTOR activation and inhibition in Tsc1-deficient aortas. Tsc fl/fl $^{1 / 1}$ yh11-CreER ${ }^{T 2} \mathrm{mT} / \mathrm{mG}$ mice were treated with tamoxifen (Tmx) or vehicle (Veh) at 1.5 weeks of age and their thoracic aortas were analyzed at various times. (A) Western blots for indicated proteins at 3 weeks with densitometry of protein bands relative to loading controls $(n=4)$. (B) Similar analyses at 12 weeks $(n=4)$. Alternatively, tamoxifen-induced mice were treated with $1 \%$ DMSO or rapamycin (RAPA) at $2 \mathrm{mg} / \mathrm{kg} / \mathrm{d}$ i.p. from 2 to 12 weeks and their thoracic aortas were analyzed. (C) Western blots for indicated proteins at 12 weeks with densitometry of phospho-proteins relative to total proteins and contractile proteins to loading controls $(n=3)$. (D) In situ examination (scale bar: 2 $\mathrm{mm}$ ) and (E) TAAD incidence. (F) H\&E stains of ascending aortas (scale bar: $100 \mu \mathrm{m}$ ) and (C) number of medial cells per cross section (cs). (H) VerhoeffVan Gieson stains of ascending aortas (scale bar: $100 \mu \mathrm{m}$ ) and $(\mathbf{I})$ number of elastin breaks per cross section ( $n=9-17$ per group, DMSO results pooled with similar results of untreated Cre-induced mice for greater statistical power). Data are represented as individual values with mean \pm SEM bars or as box-andwhisker plots with interquartile range, median, minimum, and maximum. ${ }^{*} P<0.05$; ${ }^{* *} P<0.01 ;{ }^{* *} P<0.001$ for Tmx vs. Veh or RAPA vs. DMSO by 2 -way ANOVA (A-C), Fisher's exact test (E), or $t$ test (G and $\mathbf{I})$. 
A
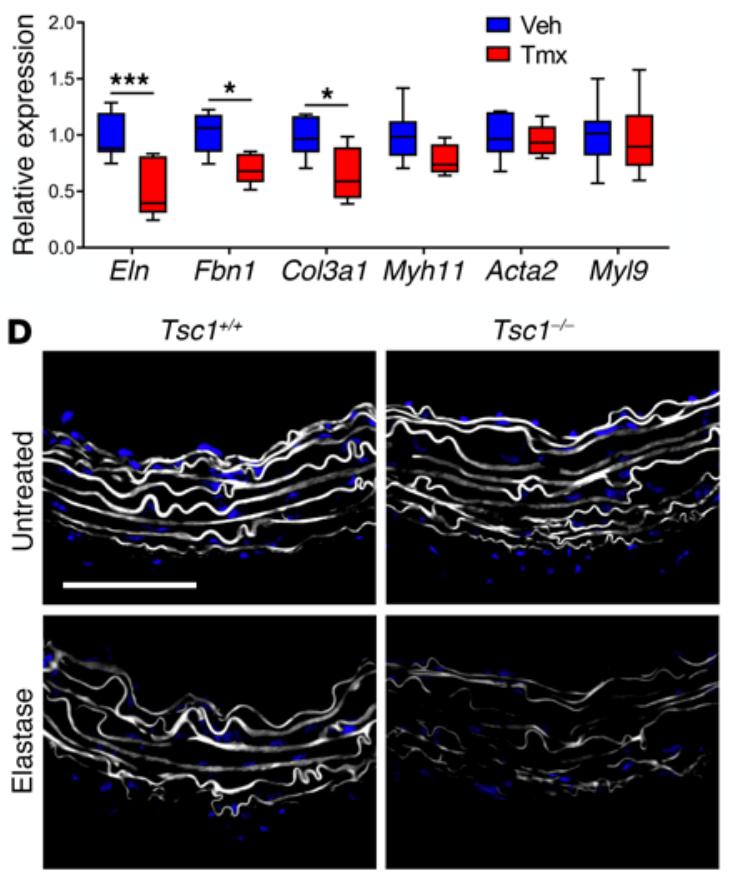

I
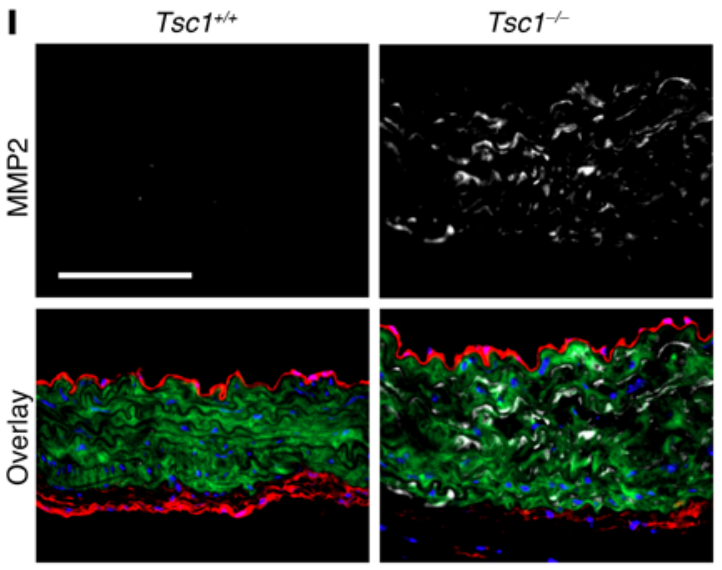

B
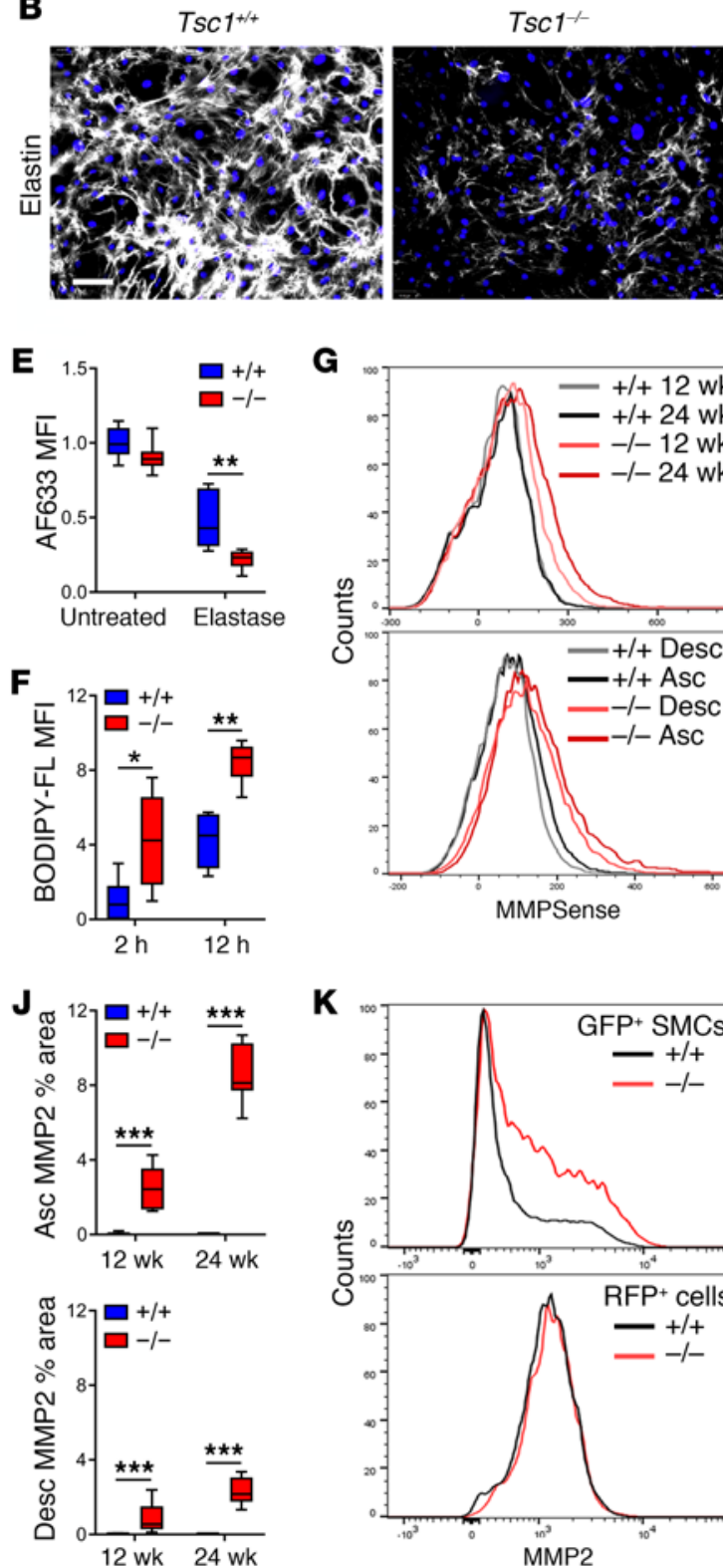

G

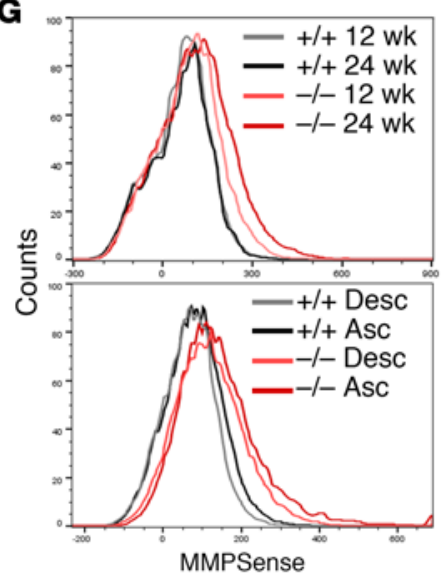

K

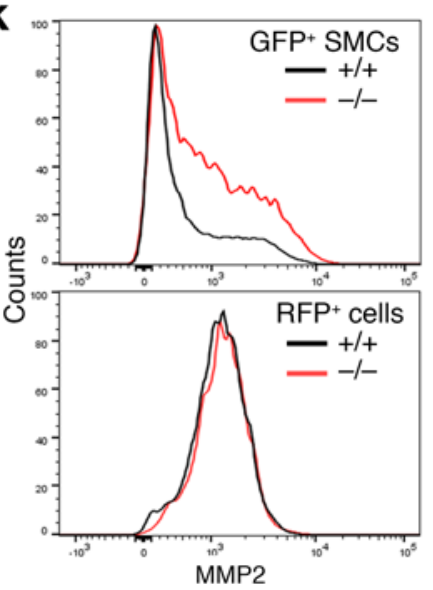

C
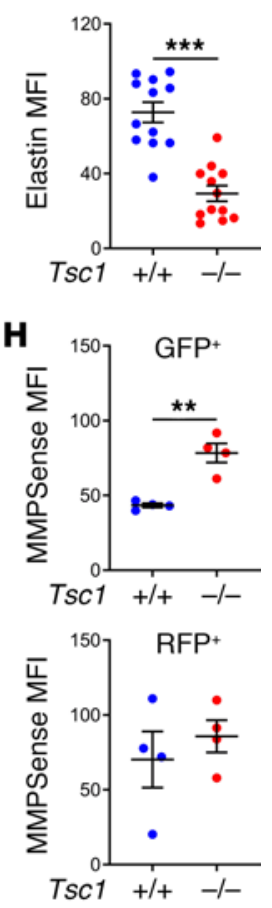

$\mathbf{L}$
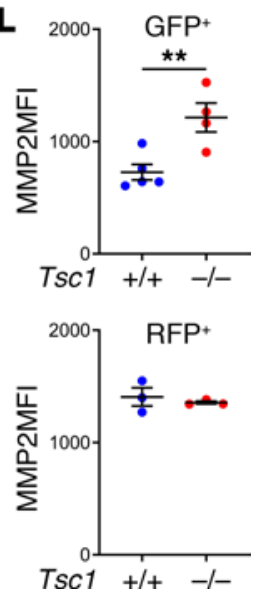

Figure 4. Tsc1 deletion in SMCs causes impaired elastogenesis and greater elastolysis. Tsc $f^{f / f l} M y h 11-C r e E R^{T 2} \mathrm{mT} / \mathrm{mG}$ mice were treated with tamox-

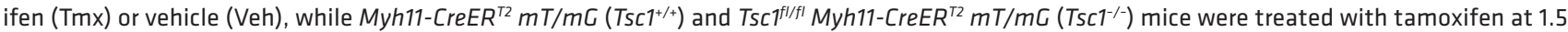
weeks of age. (A) Transcript expression in thoracic aortas of 3-week-old mice relative to Hprt ( $n=6-7)$. (B) Elastin (white) and nuclei (blue) in aortic SMC cultures from 3-week-old mice (scale bar: $100 \mu \mathrm{m}$ ) and (C) quantified as mean fluorescence intensity (MFI) ( $n=12)$. (D) Elastic fibers labeled with Alexa Fluor 633 hydrazide (AF633) in thoracic aortas of 12-week-old mice untreated or treated with elastase ex vivo (scale bar: $100 \mu \mathrm{m})$ and (E) the relative intensity was quantified $(n=7-8)$. (F) Relative intensity of cleavage products from BODIPY FL-conjugated DQ elastin incubated with thoracic aorta lysates from 24-week-old mice for 2-12 hours $(n=6)$. (G) Flow cytometry for MMPSense 645 activation by GFP+ SMCs isolated from ascending (Asc) or descending (Desc) aortas of 12- and 24-week-old mice and (H) quantified at 24 weeks $(n=4)$. (I) Immunostaining for MMP2 (white) with RFP, GFP, and DAPI overlay in ascending aortas at 24 weeks (scale bar: $100 \mu \mathrm{m}$ ) and (J) quantified in ascending and descending aortas of 12- and 24-week-old mice $(n=8)$. (K) Flow cytometry for MMP2 expression in GFP+ SMCs and RFP+ cells isolated from thoracic aortas of 24-week-old mice and (L) quantified at 24 weeks $(n=3-5)$. Data are represented as individual values with mean \pm SEM bars or as box-and-whisker plots with interquartile range, median, minimum, and maximum. Additional experimental details can be found in the supplemental methods. ${ }^{*} P<0.05 ;{ }^{* *} P<0.01 ;{ }^{* * *} P<0.001$ by 2 -way ANOVA (A, E, F, and $\mathbf{J})$ or $t$ test $(\mathbf{C}, \mathbf{H}$, and $\mathbf{L})$.

or upregulation of contractile proteins in response to serum withdrawal in cultured, Tsc1-deficient, $\mathrm{GFP}^{+}$SMCs, while repressed mTORC2 signaling was more apparent in the absence of growth factors (Supplemental Figure 4B). Administration of the mTOR inhibitor rapamycin to Tsc1-deficient mice ablated mTORC1 activ- ity without changing mTORC2 activity and increased contractile protein expression in the thoracic aorta (Figure 3C). Rapamycin therapy from 2 to 12 weeks prevented TAAD formation (Figure 3, D-I), albeit with reduced somatic growth (Supplemental Figure 5 , A-D). Treatment with rapamycin from 12 to 24 weeks, i.e., after 
A
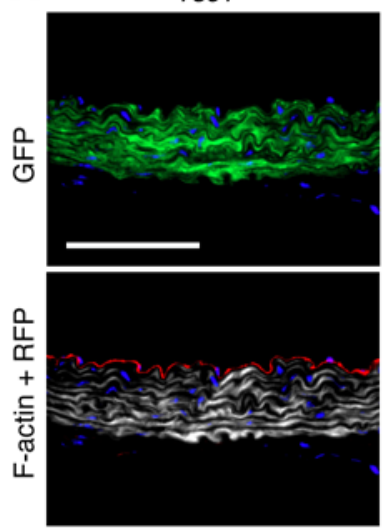

D
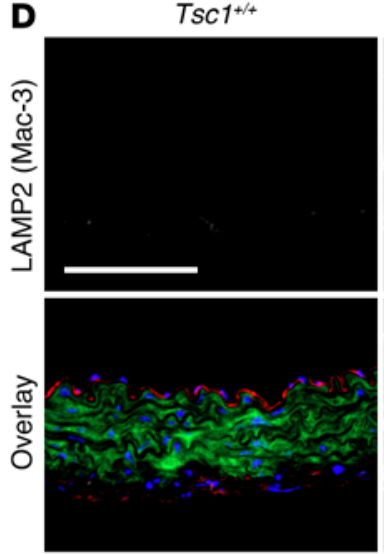

Tsc1--
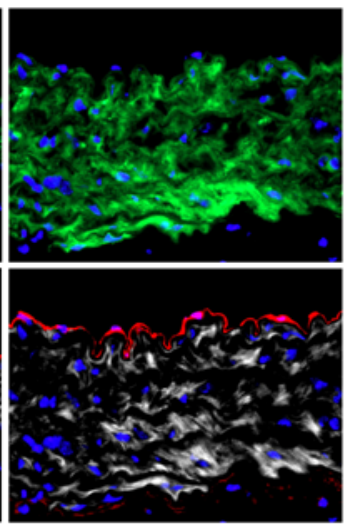

$T s c 1^{-1}$

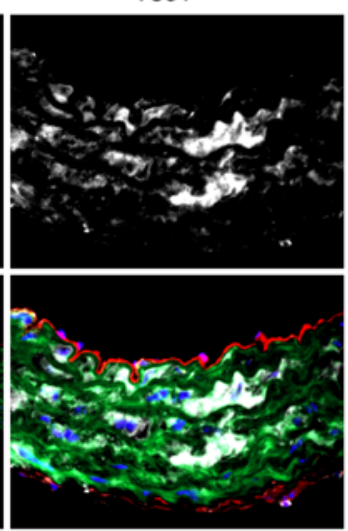

B

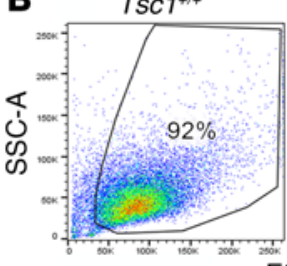

FSC-A

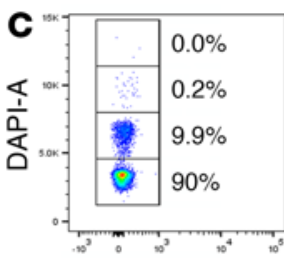

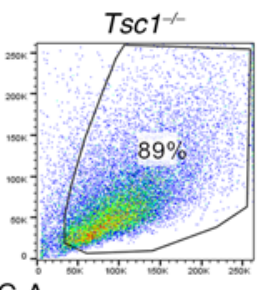

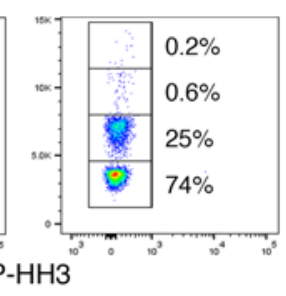

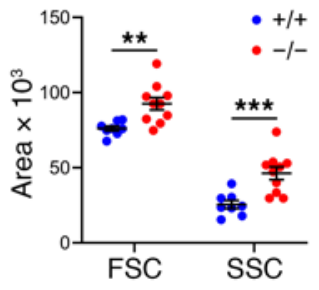
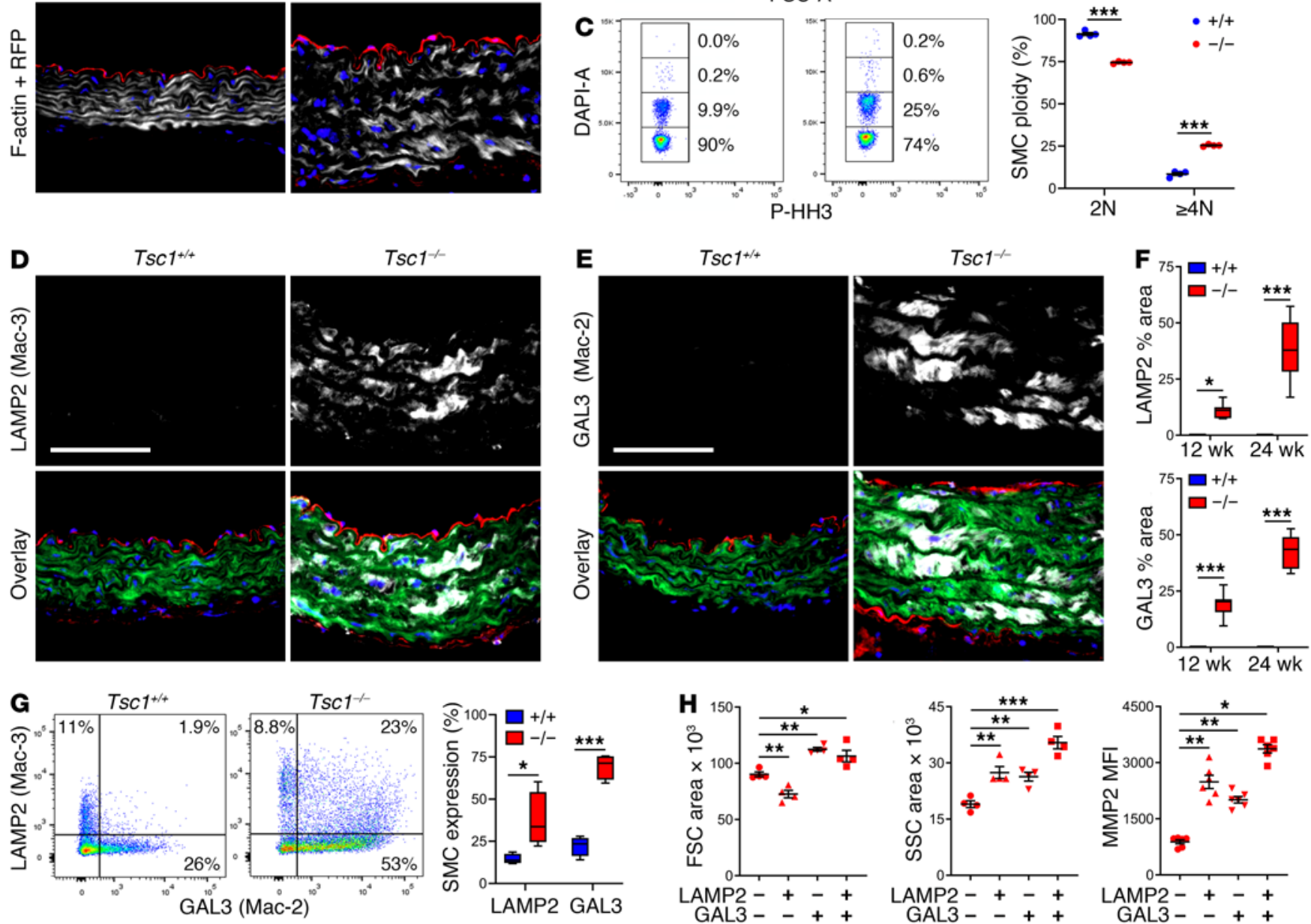

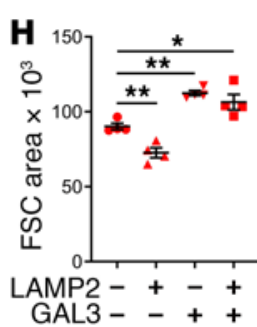

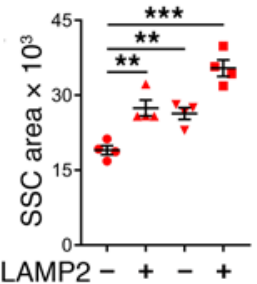

GAL3 - + + +

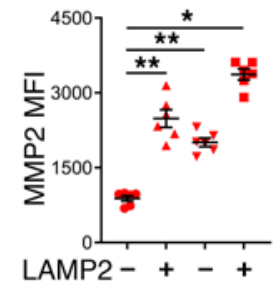

GAL3 - - + +

Figure 5. Morphologically abnormal SMCs acquire limited macrophage markers. Myh11-CreER ${ }^{T 2} \mathrm{mT} / \mathrm{mG}\left(\mathrm{Tsc} 1^{+/+}\right)$and $T s c 7^{f / / f l} \mathrm{Myh}^{11-C r e E R^{T 2}} \mathrm{mT} / \mathrm{mG}\left(\mathrm{Tsc1^{-1- }}\right)$ mice were treated with tamoxifen at 1.5 weeks and the thoracic aortas were analyzed at 24 weeks. (A) Immunofluorescence microscopy of ascending aortas identified SMCs by GFP expression (green), nuclei by DAPI labeling (blue), cells other than SMCs by RFP expression (red), and F-actin filaments by phalloidin binding (white). Scale bar: $100 \mu \mathrm{m}$. (B) Flow cytometry of enzymatically dispersed CFP+ SMCs determined forward scatter (FSC, indicator of cell size) and side scatter (SSC, indicator of cell granularity) $(n=8-10)$ and (C) fraction of diploid (2N) and polyploid ( $\geq 4 \mathrm{~N})$ nuclei by DAPI labeling in nonproliferating, phospho-histone H3-negative (p-HH3-negative) single cells $(n=5)$. (D) Immunostaining for LAMP2 (also known as Mac-3) and (E) GAL3 (also known as Mac-2) (white) with GFP, RFP, and DAPI overlay in ascending aortas (scale bars: $100 \mu \mathrm{m}$ ) and (F) quantified as percentage area ( $n=8)$. (G) Flow cytometry for intracellular expression of LAMP2 (Mac-3) and GAL3 (Mac-2) by GFP+ SMCs $(n=4)$ and (H) forward, side scatter area, and MMP2 expression by subpopulations of LAMP2/CAL3-expressing cells $(n=4-6)$. Data are represented as individual values with mean \pm SEM bars or as box-and-whisker plots with interquartile range, median, minimum, and maximum. ${ }^{*} P<0.05$; ${ }^{* *} P<0.01$; ${ }^{* *} P<0.001$ by 2 -way ANOVA (B, C, F, and $\left.\mathbf{G}\right)$ or 1 -way ANOVA (H).

disease initiation, also prevented TAAD progression (Supplemental Figure 5, E-G). Phenotypic rescue by the highly specific drug rapamycin (23) excludes mTOR-independent effects of the tuberous sclerosis complex as causes of the aortic disease (24).

Medial degeneration associates with impaired elastogenesis and greater elastolysis. We investigated if elastic fiber fragmentation results from abnormal production and/or breakdown. Because medial elastin synthesis decreases after P21 (25), we quantified the abundance of ECM transcripts in the thoracic aorta at 3 weeks
(Figure 4A). mRNA expression for Eln (encoding elastin), Fbn1 (encoding fibrillin-1), and Col3a1 (encoding type III collagen) was decreased, while that for Myh11, Acta2, and Myl9 (encoding MLC2) was unchanged, consistent with contractile protein expression at this early time. In vitro culture of aortic SMCs from 3-weekold mice also revealed decreased elastin and microfibrillar protein synthesis (Figure 4, B and C and Supplemental Figure 6, A and B). Ascending aortas from 12-week-old mice were also more susceptible to elastic fiber digestion by extrinsic enzyme (Figure 4, D 
A

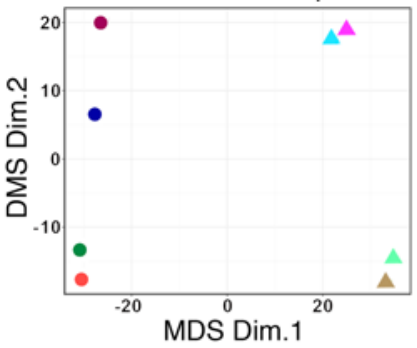

Aorta sample ID

$\mathrm{TsC}^{+/ /+} \mathrm{TsC1}^{-/-}$

- $\mathrm{i} \quad \Delta \mathrm{i}$

- ii $\Delta$ ii

- iii $\Delta$ iii

- iv $\Delta$ iv
B

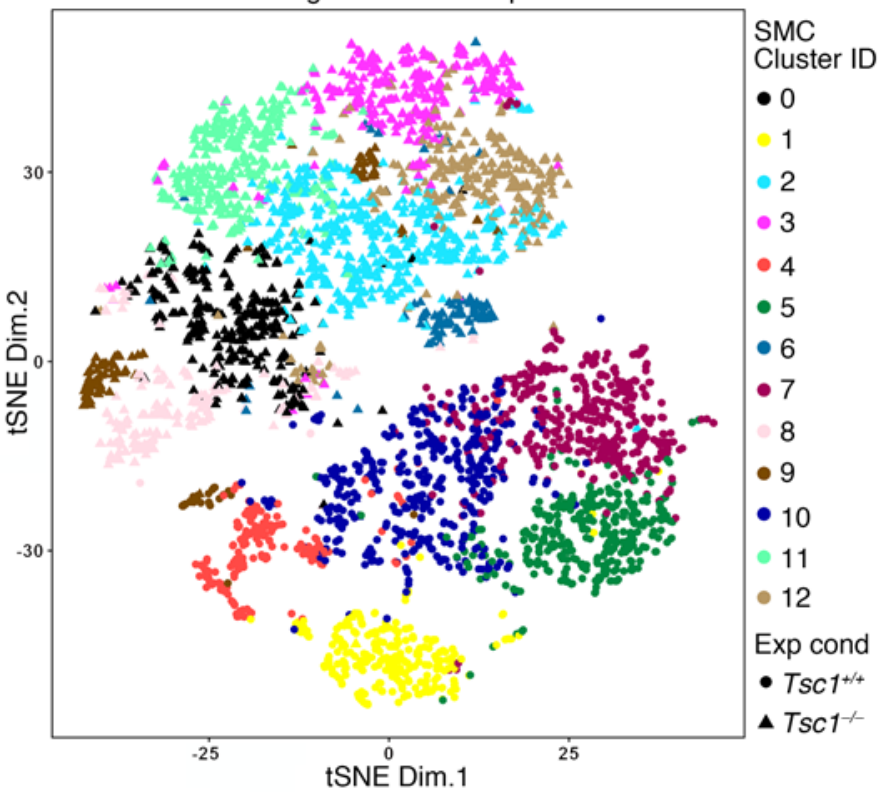

Figure 6. SMCs with downregulated contractile and synthetic molecules are characterized by markers of degradative organelles and cellular activation. Myh11-CreER ${ }^{12}$ $\mathrm{mT} / \mathrm{mG}\left(\mathrm{Tsc}^{\left.1^{++}\right)}\right.$and $\mathrm{Tsc} \mathrm{T}^{\mathrm{fl} / \mathrm{fl}}$ Myh11-CreER ${ }^{\text {T2 }} \mathrm{mT} / \mathrm{mG}\left(\mathrm{Tsc}^{-/-}\right)$mice were treated with tamoxifen at 1.5 weeks and the thoracic aortas and isolated GFP+ SMCs were analyzed at 24 weeks. (A) Multidimensional scaling (MDS) of bulk RNA-seq data $(n=4)$ shows clear delineation of $\mathrm{Tsc}^{+++}$(circular symbols) from $\mathrm{Tsc}^{1 /-}$ (triangular symbols) experimental conditions (Exp Cond). (B) T-distributed stochastic neighbor embedding (tSNE) of single-cell RNA-seq data identifying 13 cell clusters by a deep learning framework using a filtered data matrix of 2,788 cells by 8,645 genes shows clear delineation of $\mathrm{Tsc}^{1++}$ (circular symbols) from $\mathrm{Tsc}^{-1-}$ (triangular symbols) experimental conditions. (C) Heatmaps reflecting bulk and single-cell RNA expression changes for genes of interest representing contractile, synthetic, innate immunity, lysosome, other degradative organelles (autophagosomes, endosomes, and phagosomes), and cell activation (proteases, adhesion molecules, and cytokines) phenotypes. There was coordinated upregulation or downregulation of functionally related genes among $\mathrm{TsC}^{+++}$(blue header bars) versus $\mathrm{Tsc}^{-1-}$ (red header bars) aortas (numbered i-iv for each experimental condition) and SMC clusters (numbered 0-12). and E). Additionally, incubation of protein extract from thoracic aortas of 24-week-old mice increased proteolysis of extrinsic elastin (Figure 4F). We assessed elastase activity of mural cells using a protease-activatable fluorescent imaging agent administered in vivo followed by flow cytometric analysis of isolated aortic cells.
MMP activity was detected in $\mathrm{GFP}^{+} \mathrm{SMCs}$, particularly from the ascending aorta, and it increased from 12 to 24 weeks (Figure $4, \mathrm{G}$ and $\mathrm{H}$ ). In contrast, basal MMP activity by a subset of $\mathrm{RFP}^{+}$ cells was unchanged. Immunofluorescence analysis of candidate MMPs typically produced by mesenchymal cells revealed 
A

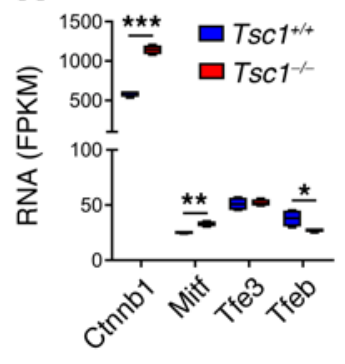

D

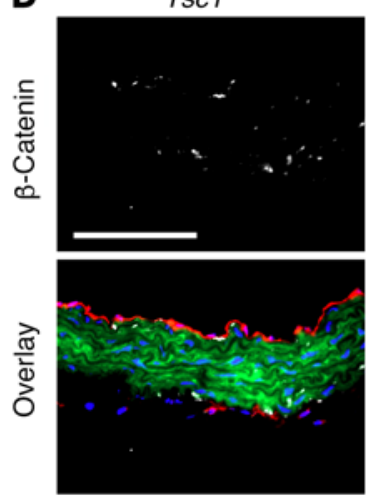

E

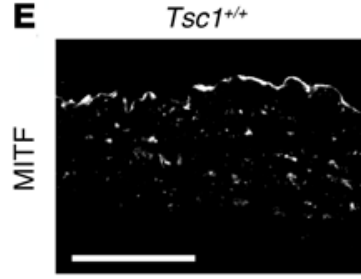

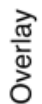

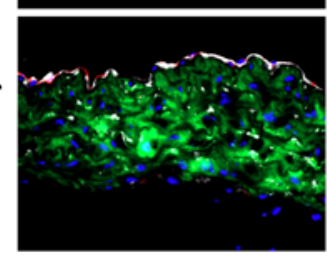

B

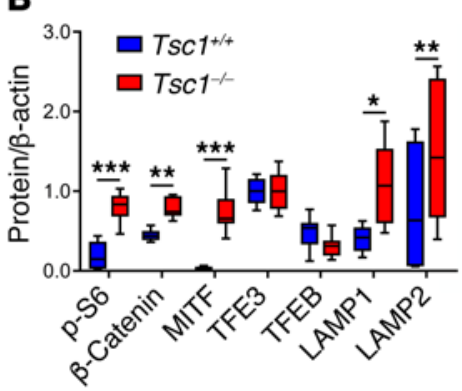

C

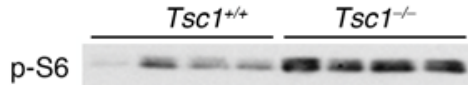

$\beta$-Catenin ------

MITF

TFE3

TFEB

LAMP1

LAMP2

$\beta$-Actin

F
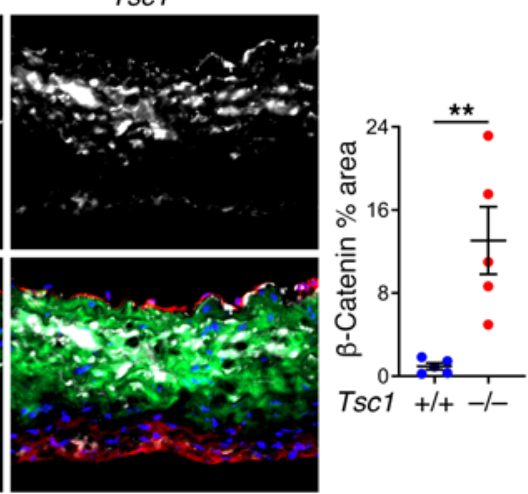

Tsc1 +1+ -1-

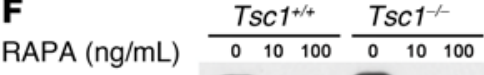

p-S6

$\beta$-Catenin

MITF

LAMP1

LAMP2

$\beta$-Actin

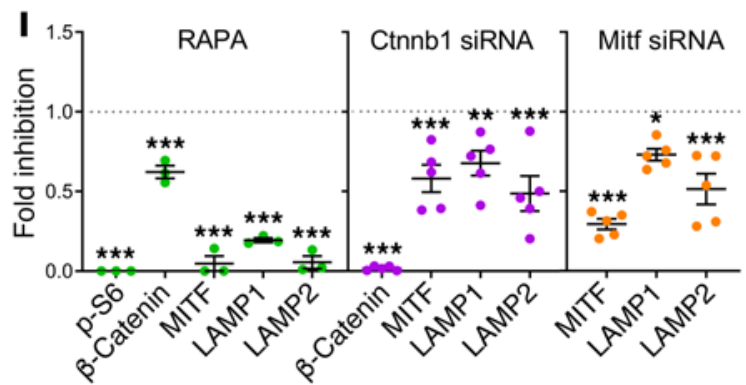

G

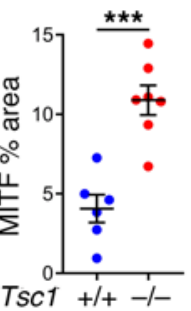

H

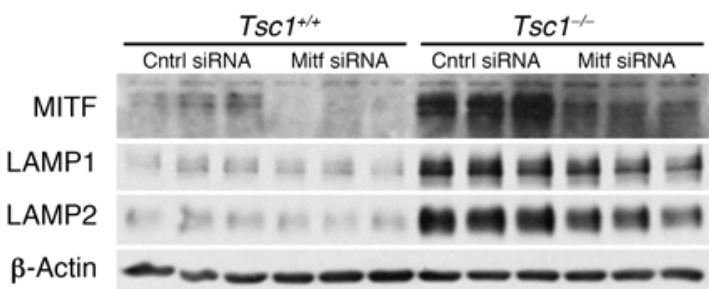

$\frac{T S C 1^{+/+}}{\text {Cntrl siRNA Ctnnb1 siRNA }} \frac{T S C 1^{--}}{\text {Cntrl siRNA Ctnnb1 siRNA }}$

$\beta$-Catenin

MITF

LAMP1

LAMP2

$\beta$-Actin
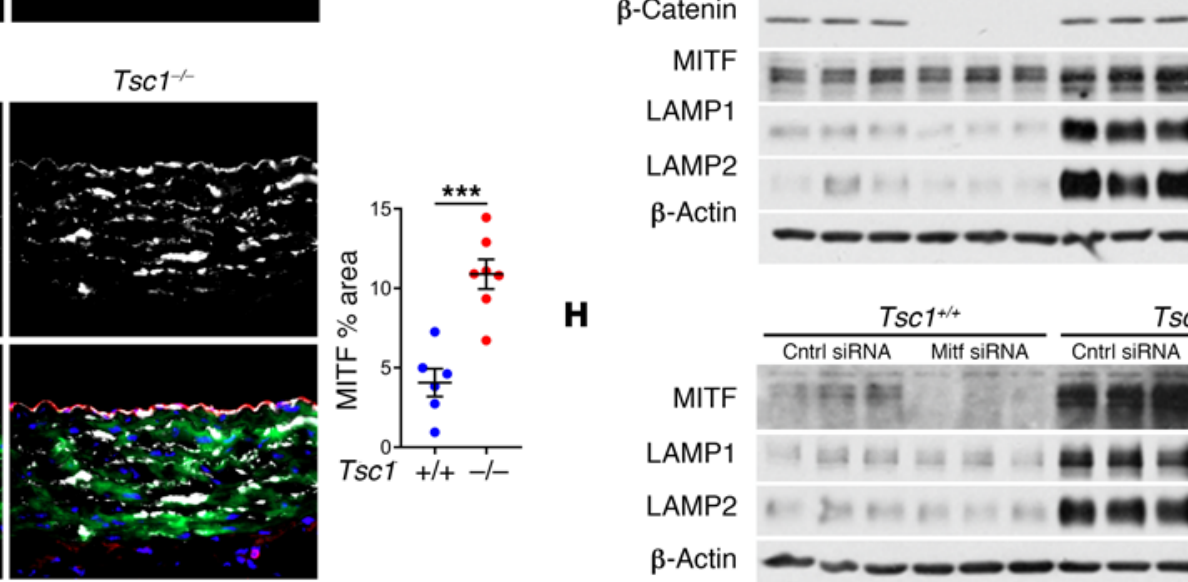

Tsc1 $+1+-1-$

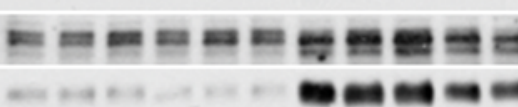

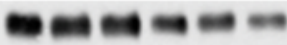
ตำ

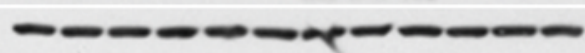

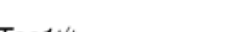

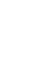



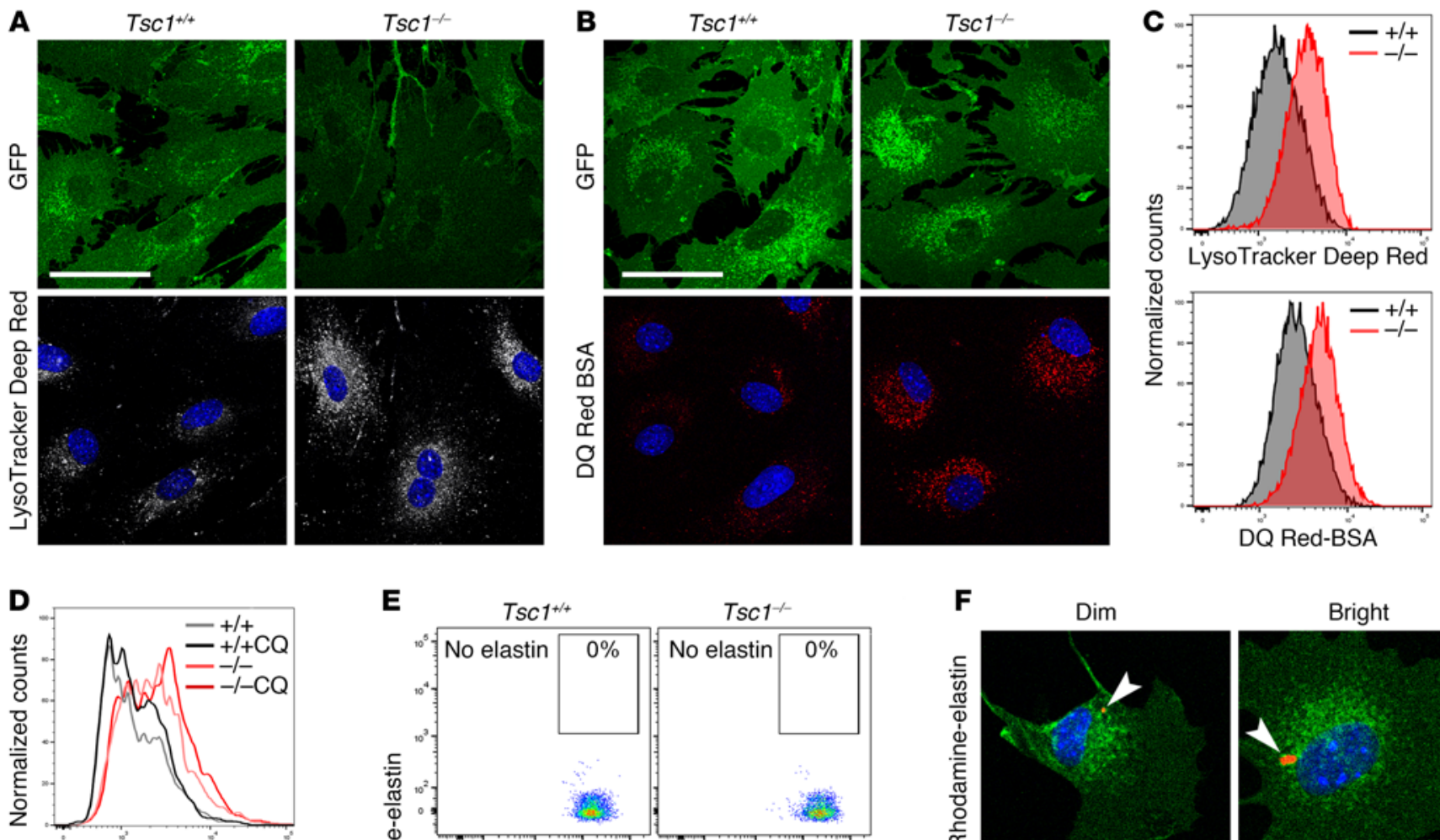

E

$\mathbf{F}$

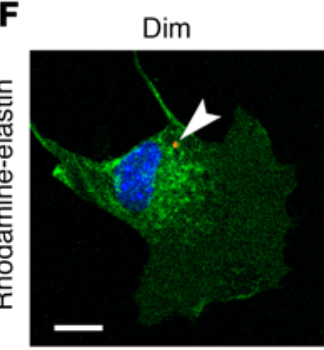

Bright

Rhodamine-Fn
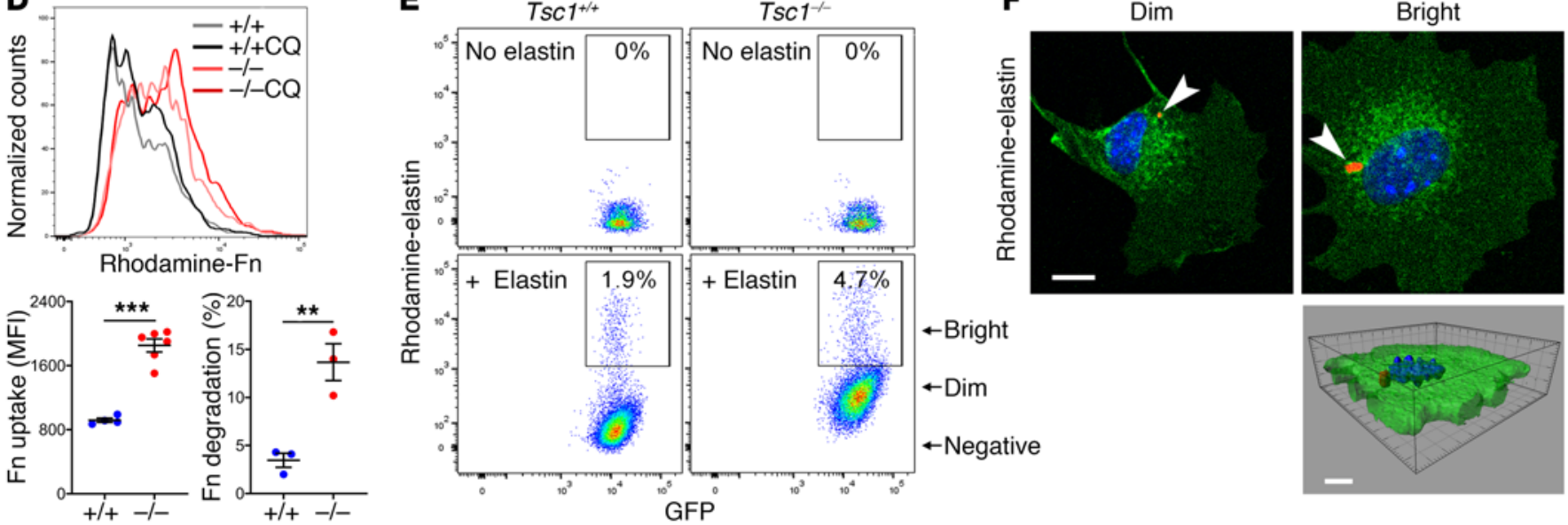

G
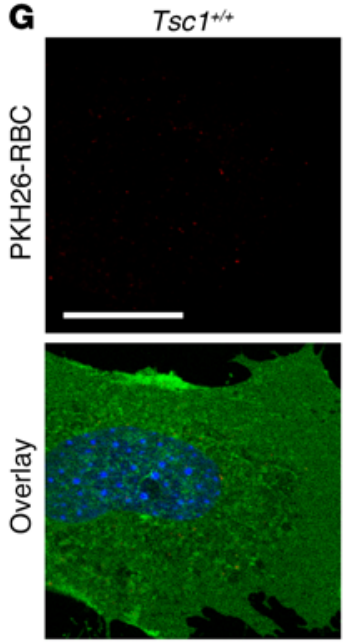

$\operatorname{Tsc1}^{-1}$

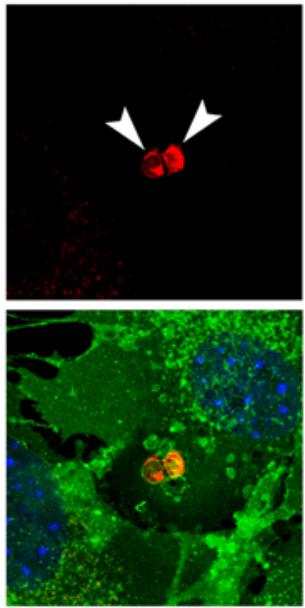

$\mathbf{H}$
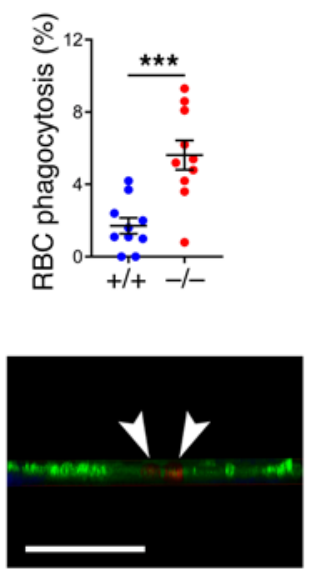

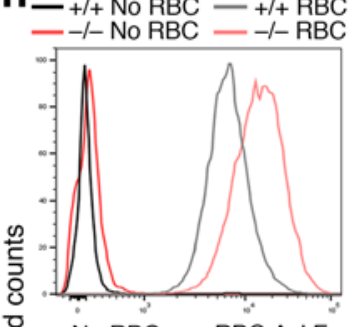

D $\stackrel{N}{N}=\mathrm{RBC} \operatorname{lgg}$ - RBC Axl Ab

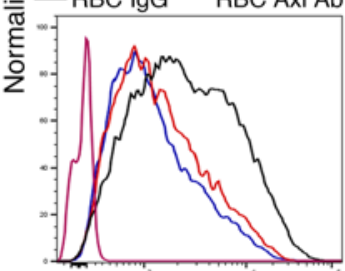

PKḦ26 Red-RBC
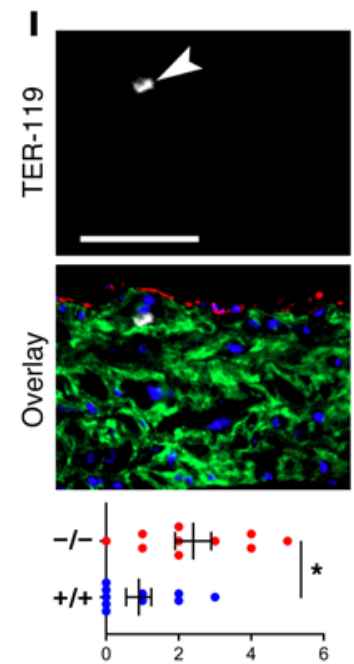

TER $-119^{+}$cells/cs 
Figure 8. SMCs gain degradative functions. SMCs were cultured from thoracic aortas of 24-week-old, tamoxifen-treated Myh11-CreER ${ }^{T 2} \mathrm{mT}$ / $m G\left(\operatorname{Tsc}^{+/+}\right)$and $T s c 7^{f / / f l} M y h 11-C r e E R^{T 2} \mathrm{mT} / \mathrm{mG}\left(\mathrm{Tsc}^{-/-}\right)$mice. Confocal microscopy of GFP+ SMCs (green) incubated with (A) LysoTracker Deep Red (white) that accumulates in perinuclear vesicles or (B) DQ Red-conjugated BSA (red) that is visualized after hydrolysis of the probe. See supplemental methods for details. Scale bars: $50 \mu \mathrm{m}$. (C) Fluorescence intensity of the cells by flow cytometry. (D) Flow cytometry of GFP+ SMCs cultured with rhodamine-labeled fibronectin (Fn) with or without chloroquine (CQ) to distinguish endocytosis-dependent uptake $(n=4-6)$ from lysosomal degradation $(n=3)$. (E) Flow cytometry of GFP+ SMCs cultured with or without rhodamine-labeled elastin and (F) confocal microscopy of cells sorted for dim or bright rhodamine fluorescence revealing elastin fragments (red, arrows) confirmed as intracellular by Z-stack image. Scale bar: $10 \mu \mathrm{m}$. (G) Confocal microscopy of GFP+ SMCs (green) cultured with PKH26-labeled, heat-damaged erythrocytes (red, arrows) confirmed as intracellular by $Z$-stack image (scale bars: $25 \mu \mathrm{m}$ ); percentage GFP+ SMCs containing erythrocytes $(n=10)$. (H) Flow cytometry of GFP+ SMCs cultured without or with erythrocytes in the presence of control IgG, AxI-neutralizing Ab, or Axl:Fc chimeric protein. (I) Immunofluorescence microscopy of ascending aorta for erythrocyte antigen, TER-119 (white, arrow) with GFP (green), RFP (red), and DAPI (blue) overlay (scale bar: $100 \mu \mathrm{m}$ ); number of medial TER$119^{+}$cells per cross section (cs) $(n=10)$. Data are represented as individual values with mean \pm SEM bars. ${ }^{*} P<0.05,{ }^{* *} P<0.01,{ }^{* * *} P<0.001$ for $\mathrm{Tsc}^{-1-}$ vs. $\mathrm{TsCl}^{+/+}$by $t$ test.

increased expression of MMP2 within the media, particularly in ascending aortas at 24 weeks (Figure 4, I and J). Increased MMP2 expression by isolated $\mathrm{GFP}^{+} \mathrm{SMCs}$ but not $\mathrm{RFP}^{+}$cells was verified by flow cytometry (Figure $4, \mathrm{~K}$ and $\mathrm{L}$ ) and zymography confirmed increased MMP2 activity in cultured cells (Supplemental Figure 6, $\mathrm{C}$ and D). Collectively, these observations demonstrate sequential changes of aberrant elastin synthesis at 3 weeks followed by greater susceptibility to proteolysis by 12 weeks plus increased induction of proteolytic enzymes between 12 and 24 weeks, all consistent with the progressive degeneration of the medial layer of the thoracic aorta found biomechanically and histologically following Tsc1 disruption.

Medial SMCs acquire a subset of macrophage markers. Given the progressive loss of contractile and synthetic functions, both defining features of SMCs, we considered possible differentiation into other cell types in the absence of cell loss. Lineage tracing by GFP expression unambiguously identified SMCs that remained within the medial layer, but they had transformed from a spindle to epithelioid morphology with increased cell size and enlarged nuclei (Figure 5A). Flow cytometric analysis of enzymatically dispersed cells confirmed the increased size as well as increased granularity and polyploidy of $\mathrm{GFP}^{+}$SMCs (Figure 5, B and C). These medial cells did not exhibit adipogenic, chondrogenic, or osteogenic phenotypes (Supplemental Figure 7A), but they did express a few macrophage antigens common to intimal SMCs in atherosclerotic plaques (14), such as lysosomal associated membrane protein-2 (LAMP2, also known as Mac-3), galectin-3 (GAL3, also known as Mac-2), and MOMA-2 (uncharacterized antigen), particularly in the ascending aorta, and this expression increased from 12 to 24 weeks (Figure 5, D-F and Supplemental Figure 7B). Absence of RFP in the media excluded infiltration or transdifferentiation into SMCs by other cell types. Additionally, $\mathrm{GFP}^{+}$medial cells did not express pan-leukocyte or several other macrophage markers, including CD45, CD11b, CD68, and F4/80 (Supplemental Figure
7, C-F). Flow cytometry verified the limited repertoire of macrophage markers by the $\mathrm{GFP}^{+}$SMCs, albeit with low expression of CD68 (Supplemental Figure 7, G and H). Further multiparameter studies revealed heterogeneous SMC populations with differing LAMP2 and GAL3 expression, thus excluding a single macrophage-like cell type (Figure 5G). LAMP2 single expressers were smaller, GAL3 single expressers were larger, and double expressers were more granular with greater production of MMP2 (Figure $5 \mathrm{H})$. Rapamycin treatment from 2 to 24 weeks of age decreased, to below basal levels, SMC size, granularity, polyploidy, and the expression of LAMP2 and GAL3, suggesting that physiological stimuli also contribute to this phenotype; rapamycin treatment for 1 week was less effective (Supplemental Figure 8, A and B). Moreover, chronic mTOR inhibition eliminated the expansion of dedifferentiated SMA ${ }^{\text {lo }}$ SMCs characterized by LAMP2 and GAL3 expression (Supplemental Figure 8, C-E). These data indicate phenotypic modulation of medial SMCs by dysregulated mTOR signaling characterized by a limited range of macrophage markers.

Phenotypically altered SMCs exhibit markers of degradative organelles. To identify additional molecules expressed by modulated SMCs with incomplete macrophage markers, we performed whole-transcriptome profiling at 24 weeks (data deposited in NCBI's Gene Expression Omnibus: GSE135177 and GSE135240). Both bulk RNA sequencing (RNA-seq) of whole thoracic aortas (Supplemental Table 3) and single-cell RNA-seq of isolated $\mathrm{GFP}^{+}$ SMCs (Supplemental Table 4) delineated experimental conditions with similar changes in selected genes of interest (Figure 6, A-C and Supplemental Figure 9). Expression of multiple contractile molecules was downregulated, as was that of many ECM molecules typically produced in the aortic wall. Notwithstanding induction of several innate immunity markers (e.g., Lamp2, Lgals3 encoding GAL3, Tlr2, and Ly96), many pan-leukocyte and myeloid lineage markers (e.g., Ptprc encoding CD45, Cd56, Itgam encoding CD11b, Lyz2, Ly6c2, Csf1r, Ccr2, Cx3cr1, Fcgr2b, and Fcgr3) were absent in $\mathrm{GFP}^{+}$SMCs, although they were expressed by a subset of contaminating $\mathrm{GFP}^{-}$cells (Supplemental Figure 10). Besides Lamp2, there was also upregulation of other lysosomal genes (e.g., Lamp1, Cd63, Ctsd, Ctsl, AtpvOb, Atp6ap1, Hexa, and Npc2) as well as markers of other organelles that contribute to intracellular digestion, such as autophagosomes (e.g., Gabarap, Gabarapl1, Map1lc3b, and Sqstm1), endosomes (e.g., Rab7), and phagosomes (e.g., Axl and Gas6). Induction of cytokine, chemokine, adhesion molecule, and protease genes (e.g., Spp1 encoding osteopontin, Cx3cl1, Vcam1, and Mmp2) suggested SMC activation. Identification of larger numbers of discriminating genes by deep learning that clustered similar cells showed similar phenotypic changes in SMCs of diverse origin, that is, with or without neural crestassociated transcription factors (26), and independent of cell damage, namely, with or without induction of mitochondrial RNA (27) (Supplemental Figure 11). Gene ontology analysis identified "proton-transporting V-type ATPase complex" (which acidifies the lysosomal lumen) in bulk RNA-seq data and "membrane-bounded organelles" in single-cell RNA-seq data among enriched terms characterizing the differences across experimental conditions (Supplemental Tables 5 and 6). Additionally, "proteolysis" and "catabolic process" were highly ranked in SMC clusters characterized by increased lysosome-related molecules (Supplemental 

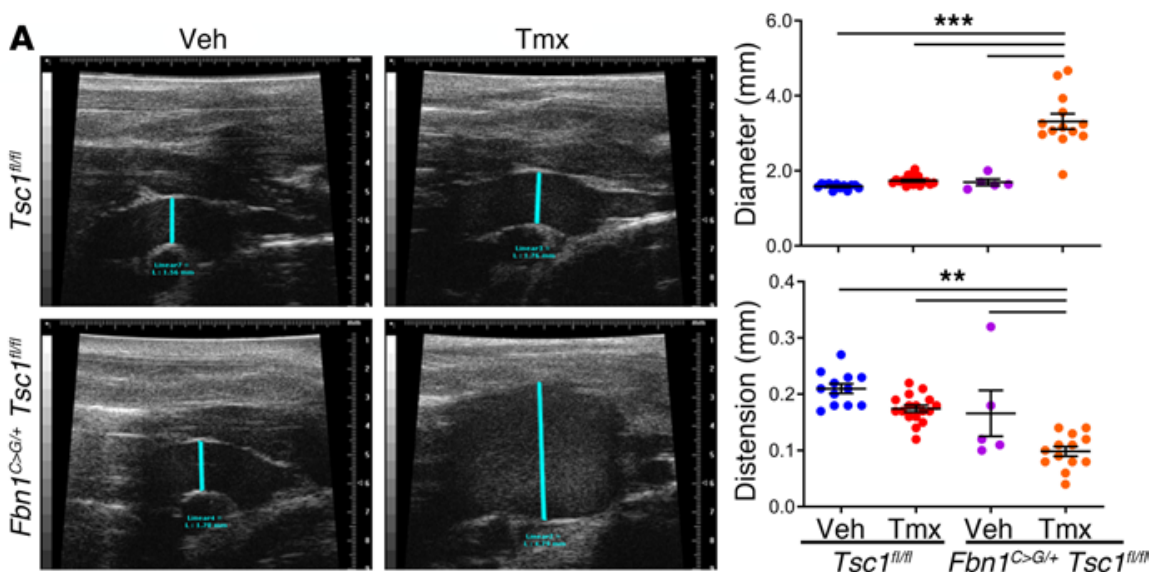

B
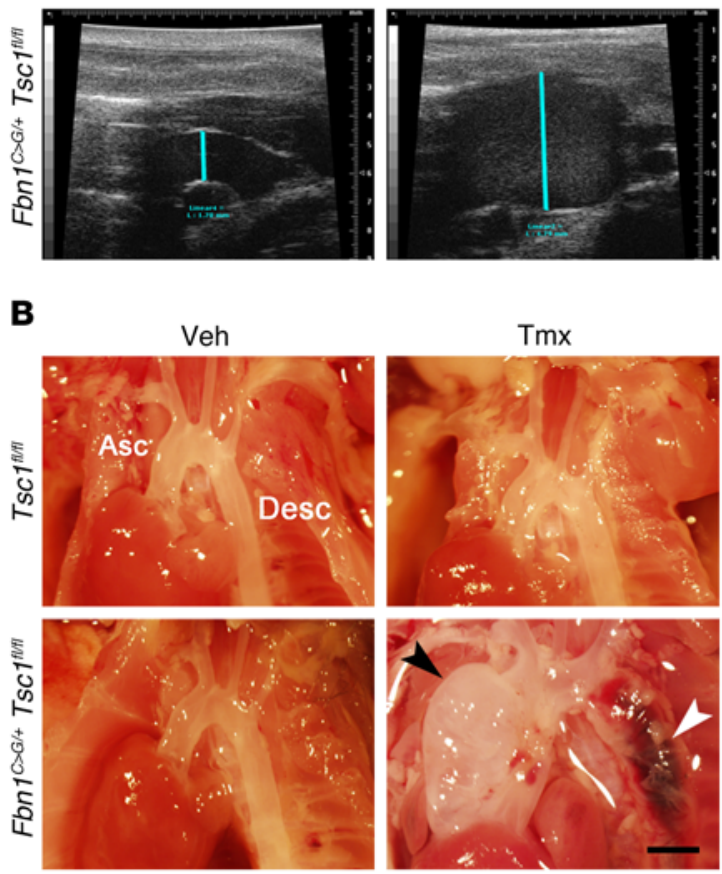

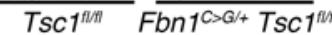
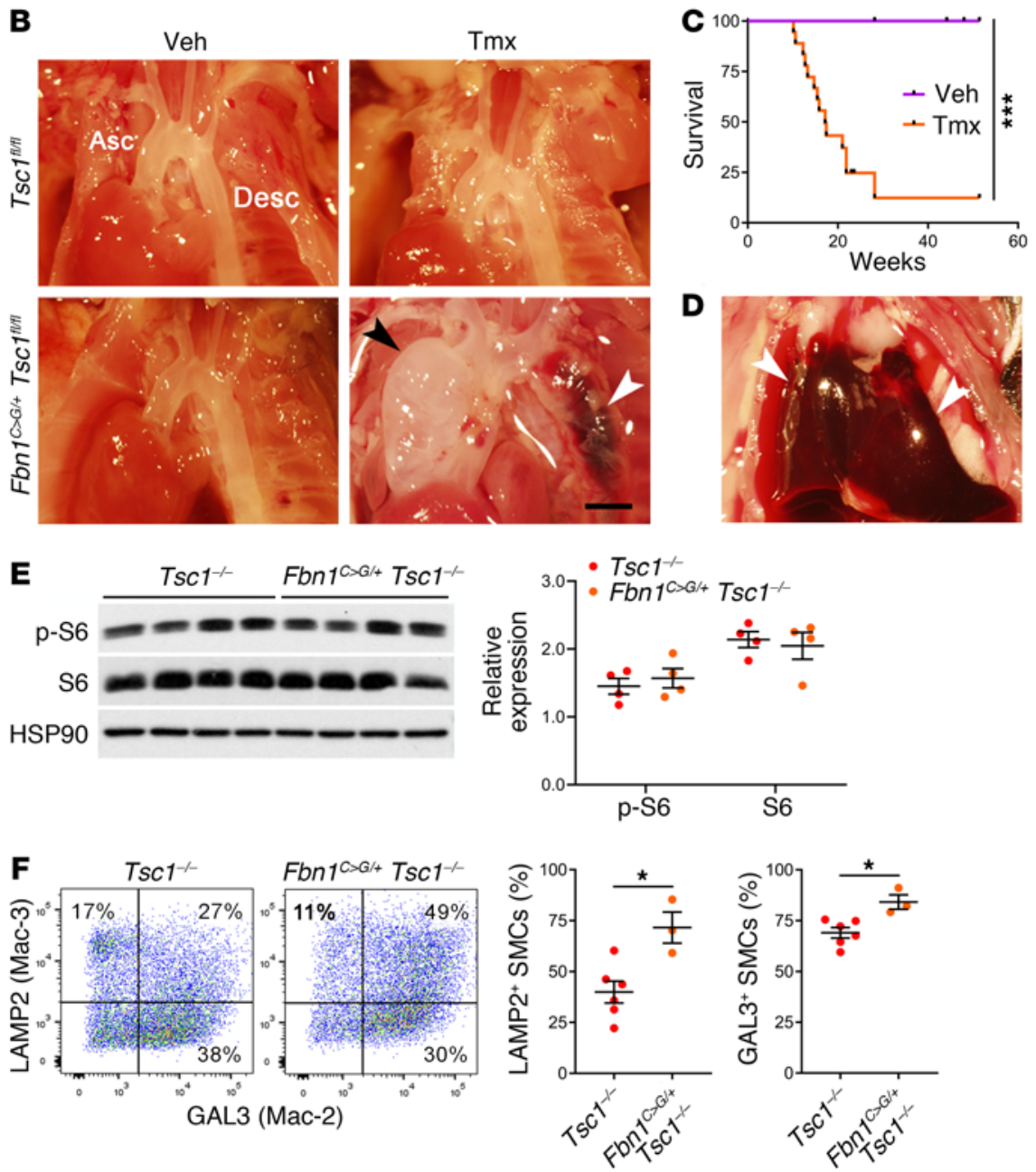

Figure 9. Degradative SMCs exacerbate TAAD in a murine model of Marfan syndrome. TsC $7^{f l / f l}$ Myh11-CreER ${ }^{T 2} \mathrm{mT} / \mathrm{mG}\left(\operatorname{Tsc}^{f l / f l}\right)$ and Fbn $7^{\mathrm{C1039C/+}}$

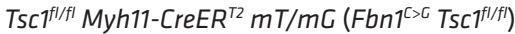
mice were treated with tamoxifen (Tmx) or vehicle (Veh) at 1.5 weeks of age and their thoracic aortas examined at 12 weeks. (A) Ultrasound examination of ascending aortas (blue line) and measurements of in vivo diameter and distension ( $n=$ 5-17). (B) In situ examination of ascending (Asc) and descending (Desc) aortas (scale bar: $2 \mathrm{~mm}$ ) showing aneurysm (black arrow) and dissection (white arrow). (c) Survival of $F b n 1^{c>c} T s c f^{f l / f l}$ mice treated with tamoxifen or vehicle $(n=17-18)$ and (D) hemopericardium (arrows) in animal with premature death. (E) mTOR signaling in thoracic aortas of tamoxifen-treated mice by Western blot and expression of phospho-S6 and $\mathrm{S} 6$ relative to HSP9O $(n=4)$. (F) Flow cytometry for LAMP2 (Mac-3) and GAL3 (Mac-2) expression by GFP+ SMCs in tamoxifen-treated mice $(n=3-6)$. Data are represented as individual values with mean \pm SEM bars. ${ }^{*} P<0.05$; ${ }^{*} P<0.01$; ${ }^{* * *} P<0.001$ by 2-way ANOVA (A and E), log-rank test (C), or $t$ test $(\mathbf{F})$.

$\beta$-catenin) in addition to multiple lysosomeand autophagosome-associated genes. Of the microphthalmia family of transcription factors known to regulate lysosomal biogenesis, Mitf and Tfe3 correlated with lysosome-related genes and Ctnnb1, whereas Tfeb correlated with smooth muscle contractile markers. Bulk RNA-seq analysis of aortic tissue revealed increased Ctnnb1 and Mitf, unchanged Tfe3, and decreased $T f e b$ expression after $T s c 1$ disruption (Figure 7A). Correspondingly, $\beta$-catenin and MITF, but not TFE3 or TFEB protein expression, was increased in vivo by Western blot (Figure $7, \mathrm{~B}$ and $\mathrm{C}$ ). Expression of $\beta$-catenin and MITF was localized to medial SMCs by immunofluorescence analysis (Figure 7, D and E). Such differences in expression were also noted in cultured SMCs, and MITF translocated to the nucleus following Wnt activation in vitro (Supplemental Figure 13). Levels of $\beta$-catenin, MITF, and lysosomal membrane proteins in cultured SMCs depended on mTOR activity (Fig-

Figure 12). Thus, transcript expression supports the concept of an SMC phenotype characterized by markers of degradative organelles that is neither part of the contractile-to-synthetic spectrum nor a result of transdifferentiation of SMCs into conventional macrophages.

An mTOR/ $\beta$-catenin/MITF pathway in SMCs regulates lysosomal biogenesis. We searched for genes that may be linked to lysosomal biogenesis in SMCs by coexpression analysis of the single-cell RNA-seq data (Supplemental Table 7). Lamp2 showed high correlation to the Wnt signaling effector Ctnnb1 (encoding ure 7F). Furthermore, knockdown of Ctnnb1 and Mitf decreased expression of lysosomal membrane proteins (Figure 7, G-I). These results indicate a coordinated regulation of lysosomal biogenesis in degradative SMCs by mTOR, $\beta$-catenin, and MITF.

Medial SMCs gain degradative functions. To determine if the altered phenotype enriched in markers of degradative organelles had functional consequences, we further characterized properties of isolated SMCs in vitro. Increased numbers of acidic vesicles extended peripherally from their normal perinuclear location as documented by an acidotropic tracker dye (Figure 8A), consistent 
A
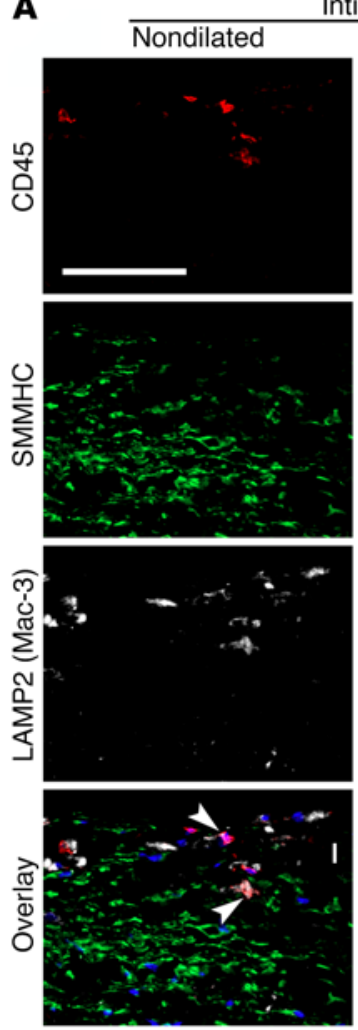

D
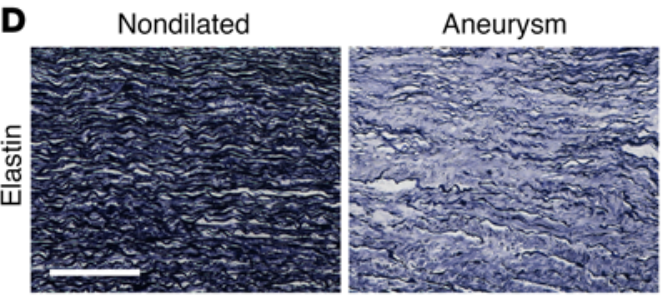

E
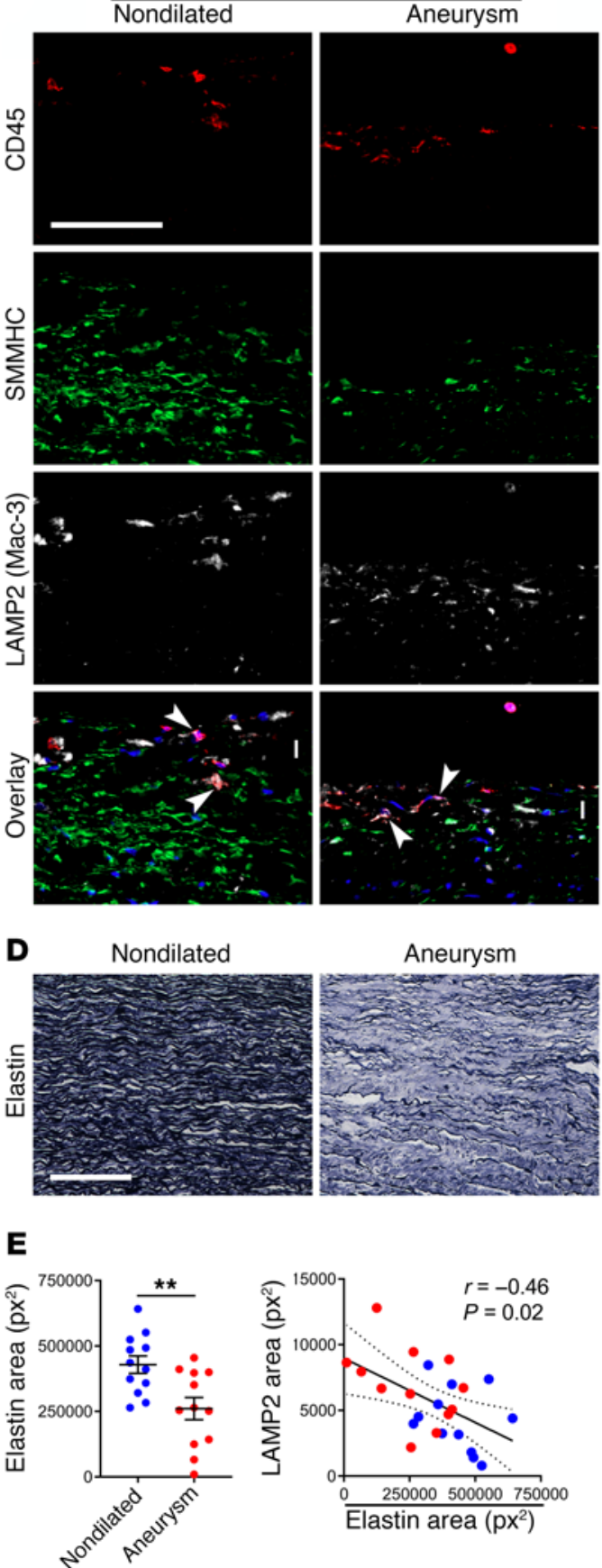

Aneurysm
B

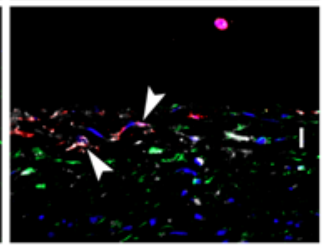

$\mathbf{F}$

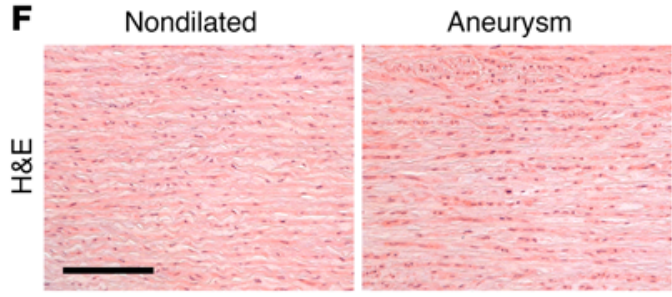

G

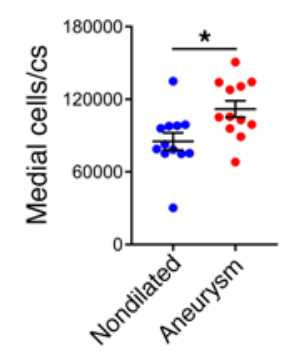

Media
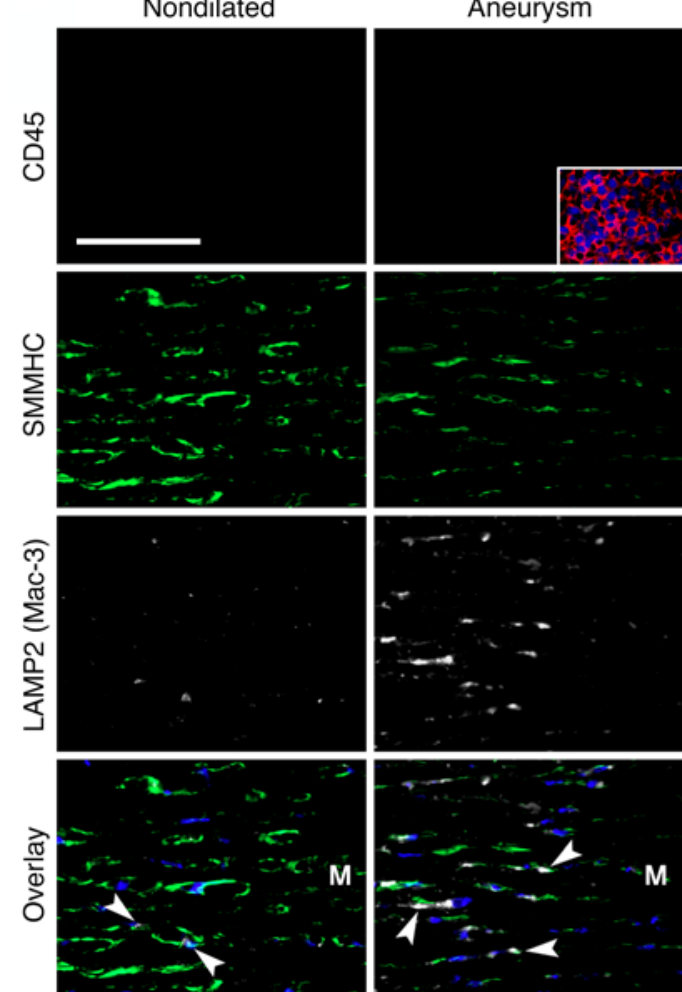

M

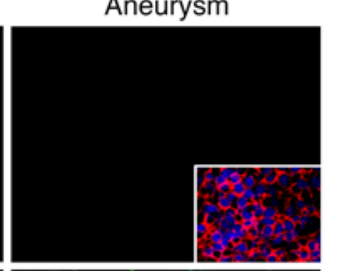

$\sum^{5}$
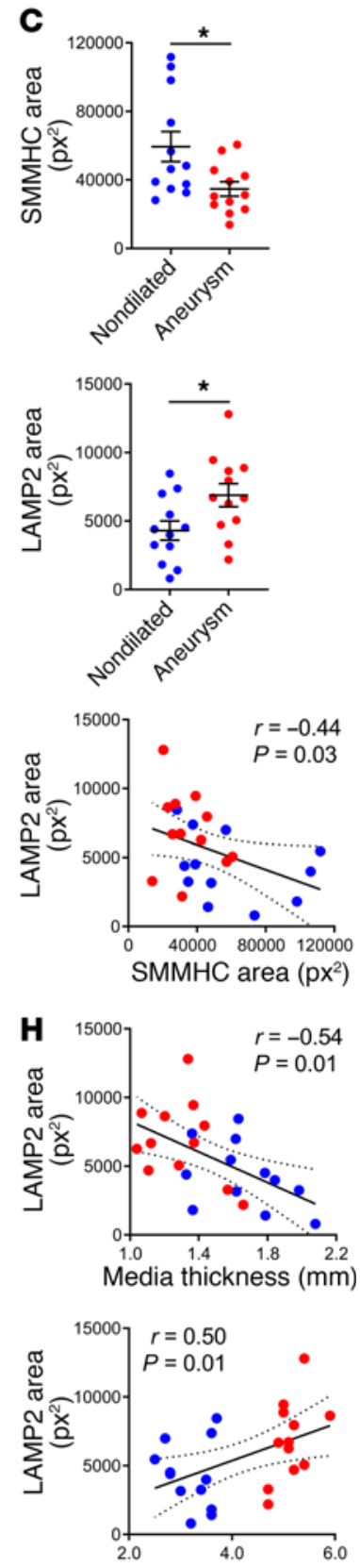

Aorta diameter $(\mathrm{cm})$

Figure 10. Degradative SMCs in human TAAD. Ascending aortas were procured from 24 subjects undergoing aortic surgery (Aneurysm) or from organ donors (Nondilated). (A and B) Immunofluorescence analysis for CD45 (red), SMMHC (green), LAMP2 (also known as Mac-3; white), and overlays with DAPI-labeled nuclei (blue) showing LAMP2+ leukocytes (arrows) in the intima (I) and LAMP2 ${ }^{+}$SMCs (arrows) in the media (M); inset of spleen positive control (scale bars: $100 \mu \mathrm{m})$. (C) Expression of SMMHC and LAMP2 $(n=12)$ and correlation of SMMHC to LAMP2 $(n=24)$. px², pixels squared. (D) Verhoeff stain of aortic media for elastin (scale bar: $200 \mu \mathrm{m})$. (E) Elastin expression $(n=12)$ and correlation of elastin loss to LAMP2 $(n=24)$. (F) H\&E stain of media (scale bar: $200 \mu \mathrm{m})$. (G) Number of medial cells per cross section $(\mathrm{cs})(n=12)$ and correlation of medial cells to LAMP2 $(n=24)$. (H) Correlation of media thickness and aorta diameter to LAMP2 $(n=24)$. Data are represented as individual values with mean \pm SEM bars or linear regression lines. ${ }^{*} P<0.05$, ${ }^{*} P<0.01$ by $t$ test $(\mathbf{C}, \mathbf{E}$, and $\mathbf{G})$ or Spearman's test for $r$ correlation coefficient $(\mathbf{C}, \mathbf{E}, \mathbf{G}$, and $\mathbf{H})$.

with similar in situ findings of more numerous and widespread organelles by electron microscopy. Increased lysosomal function was determined with conjugated bovine serum albumin in which self-quenched fluorescence is detectable only after hydrolysis (Figure 8B). Differences in lysosomal number and function by confocal microscopy were verified by flow cytometry (Figure 8C). Receptor-mediated endocytosis of fibronectin by cultured SMCs is well established (28), and this assay of ECM uptake revealed both greater accumulation and degradation of labeled fibronectin (Figure 8D). In contrast, long-term treatment with rapamycin in 
vivo decreased SMC uptake and breakdown of fibronectin (Supplemental Figure 14A). SMCs also displayed increased phagocytosis of labeled, insoluble elastin fragments in coculture (Figure $8 \mathrm{E})$. Although most cells accumulated small $(<1 \mu \mathrm{m})$ elastin particles within perinuclear vesicles, a minority characterized by high rhodamine fluorescence also contained relatively large $(>1$ $\mu \mathrm{m}$ ) elastin inclusions (Figure $8 \mathrm{~F}$ ). Because mural hemorrhage and medial cell death may occur with TAAD progression, we also tested for phagocytosis of labeled, heat-damaged cells and found increased efferocytosis of erythrocytes, mononuclear cells, and damaged SMCs by viable SMCs (Figure 8G and Supplemental Figure 14, B and C). The vacuoles containing elastin particles or damaged erythrocytes were lined by lysosomal membrane proteins, identifying the structures as phagolysosomes (Supplemental Figure 14, D and E). We defined a functional role for Axl and Gas6, both of which were increased in RNA-seq analyses, using neutralizing Axl antibodies and soluble Axl-Fc chimeric proteins (Figure $8 \mathrm{H})$. Interestingly, phagocytosis of erythrocytes may occur in vivo in the absence of overt mural bleeding, as we documented occasional TER $-119^{+}$cells within or contiguous to medial $\mathrm{GFP}^{+} \mathrm{SMCs}$ of nondissected thoracic aortas (Figure 8I). The finding of infrequent, large cytoplasmic vacuoles containing amorphous material by electron microscopic examination of tissue sections suggested in vivo SMC phagocytosis (Supplemental Figure 14F). These observations establish that Tsc1-deficient SMCs are more efficient at uptake and degradation of ECM and cellular debris.

mTOR-transformed SMCs are deleterious in diverse models of aortic disease. Because degradative SMCs could promote either reparative or pathological processes (e.g., by removing ECM debris or breaking down intact ECM), we investigated consequences of mTOR hyperactivation in an experimental model of mild TAAD attributable to a clinically relevant mutation of Fbn1. We previously reported activation of $\mathrm{mTOR}$ signaling in the thoracic aortas of $\mathrm{Fbn}^{\mathrm{C1039G/+}}$ mice (29) and found increased LAMP2 ${ }^{+}$and GAL3 ${ }^{+}$ SMCs late in the disease course (Supplemental Figure 15). In contrast with controls, $F b n 1^{\mathrm{C1039G} /+} T s c 1^{f l / f l}$ Myh11-CreER ${ }^{T 2} \mathrm{mT} / \mathrm{mG}$ mice induced with tamoxifen at 1.5 weeks presented markedly enlarged ascending aortas by ultrasound and gross examination at 12 weeks of age (Figure 9, A and B), well before that in $\mathrm{Fbn}^{\mathrm{C} 1039 \mathrm{G} /{ }^{+}}$mice (29). Strikingly, almost all compound mutants died from aortic rupture between 12 and 24 weeks (Figure 9, C and D). Greater disease severity was associated with $\mathrm{mTOR}$ activation and an increased frequency of LAMP2/GAL3 ${ }^{+}$SMCs (Figure 9, E and F). We also investigated effects of a degradative SMC phenotype on aortic atherosclerosis by crossing Tsc1 mutants with $A p o E^{-/-}$mice, because hyperlipidemia is reported to drive transdifferentiation of SMCs into macrophages (30). To avoid plaque disruption by aortic dissection, we delayed the administration of tamoxifen to 7 weeks of age, or 1 week prior to the initiation of a high-fat, high-cholesterol diet, that resulted in a low incidence of TAAD (17\%) at 24 weeks, with dissected vessels excluded from analysis. Conditional disruption of $T s c 1$ increased lipid accumulation within the aorta that localized to intimal cells as well as medial SMCs adjacent to and remote from intimal plaques (Supplemental Figure 16, A and B). Medial and, particularly, intimal $\mathrm{GFP}^{+} \mathrm{SMCs}$ expressed the lysosomal marker LAMP2 but not the pan-leukocyte marker CD45 on confocal microscopy (Supplemental Figure 16, C and D). We con- firmed by transcriptional profiling and flow cytometry that fatemapped SMCs from atheromatous aortas of tamoxifen-treated ApoE ${ }^{-/-}$Myh11-CreER ${ }^{T 2} \mathrm{mT} / \mathrm{mG}$ control mice did not express macrophage markers except for lysosome-associated molecules (Supplemental Figures 17 and 18). These outcomes support pathogenic effects of mTOR activation in SMCs on aortic disease beyond models of tuberous sclerosis.

SMCs with degradative phenotype occur in human TAAD. Finally, to determine if these many experimental findings are relevant to human disease, we analyzed both aneurysmal and nondilated ascending aorta specimens from 24 patients. The subject groups differed for sex, age, bicuspid aortic valve, and dyslipidemia (Supplemental Table 8). Among other cell types, CD45+ leukocytes expressed LAMP2 (Mac-3) within the inflamed intima, while $\mathrm{SMMHC}^{+}$SMCs expressed LAMP2 within the bland media (Figure 10, A and B). Medial SMCs of aneurysmal aortas expressed greater LAMP2 and less SMMHC than nondilated controls (Figure 10C). An inverse correlation of LAMP2 to SMMHC expression further suggested increased lysosomes in dedifferentiated SMCs. Additionally, aneurysms displayed characteristic elastin fragmentation and LAMP2 expression correlated with loss of elastin (Figure 10, D and E). As previously reported (3), small thoracic aortic aneurysms had more medial cells than referent nondilated vessels and LAMP2 expression correlated with the number of medial cells (Figure 10, F and G and Supplemental Table 8). LAMP2 expression also correlated to medial thinning and ascending aorta dilatation (Figure 10H). Furthermore, mTOR signaling was increased in TAAD, and SMCs with detectable mTOR activity had increased expression of LAMP2 (Supplemental Figure 19). With a caveat of potentially confounding clinical differences among subject cohorts, medial SMCs with a degradative phenotype manifest in human TAAD, thus supporting a pathological role.

\section{Discussion}

Our findings elucidate a role of dramatic SMC modulation in TAAD. SMCs with mTOR-dependent proteolytic and phagocytic activity drive TAAD formation. Moreover, these degradative SMCs do not fit within the canonical spectrum of contractileto-synthetic phenotypic modulation typical of many arterial diseases and their upregulation of lysosomal markers does not imply transdifferentiation into conventional macrophages. Rather, these proliferative SMCs exhibit a unique subset of degradative properties that compromise aortic medial properties and function, thereby rendering the wall vulnerable to dilatation and dissection. Hence, promoting SMC proliferation without maintaining appropriate function will not prevent TAAD. Indeed, this could worsen medial degeneration and exacerbate TAAD.

We used a model of conditional Tsc1 disruption to test a hypothesis focused on altered SMC function, not to recreate a rare aortic disease of children with heterozygous germline mutations affecting the tuberous sclerosis complex $(8,9,11)$. Importantly, this model revealed that mTORC1 hyperactivation leads to increased proliferation of medial SMCs and progressive TAAD. Medial degeneration and luminal dilatation initially affect the ascending aorta, and then extend distally with age. Differences in mechanical stresses or in embryological origins of SMCs may dictate segmental vulnerability $(31,32)$. Diminished elastic fiber 
integrity and associated loss of elastic energy storage initiates aortic remodeling in our model and is multifactorial with distinct temporal onsets: (a) aberrant elastic fiber synthesis, (b) enhanced susceptibility to proteolysis, (c) diminished SMC contractility reducing stress shielding of the ECM, (d) greater protease production, and (e) degradative organelle biogenesis with increased macromolecule clearance. In addition to the manifestation of spontaneous disease, despite the lower blood pressure, these abnormalities exacerbate aortopathy resulting from other causes, such as fibrillin-1 mutation.

SMC proliferation is recognized as contributing to occlusive but not aneurysmal arterial disease. Conspicuous acellular areas of the aneurysmal media have long been recognized in end-stage disease and it is intuitive to relate cell death to medial thinning and mural rupture (2). Yet, SMC loss also occurs in nondilated aortas (33) and foci of SMC hyperplasia are found in TAAD (6, $7,34)$. Few studies have counted aortic SMCs. Consistent with our previous report (3), the present data confirm that medial area can increase in thin-walled aneurysmal thoracic aortas and SMC density can remain unchanged, hence revealing increases in the number of SMCs per cross section and thus hyperplasia unless due to cell migration. Similar findings of preserved SMC density in TAAD have been reported independently (15), while others report $58 \%-150 \%$ increases $(34,35)$ or $23 \%-36 \%$ decreases $(36$, 37 ), with variations across studies perhaps highlighting focal differences. Moreover, SMC death does not necessarily cause TAAD. Selective SMC apoptosis in mice leads to aortic wall thinning without outward remodeling (38); aortic allografts in nonimmunosuppressed patients function as valved conduits without aneurysmal enlargement even with inevitable SMC death (39). These observations suggest that viable cellular participants are required to remodel extant ECM and allow aortic dilatation. Although early induction of medial cell hyperplasia in our model temporally coincides with certain dysfunctional properties (e.g., decreased elastin synthesis at 3 weeks of age), the incremental increase in degradative markers from 12 to 24 weeks implies chronic indicators of and stimuli for phenotypic modulation. Additionally, the limited efficacy of short-term versus long-term rapamycin treatment in reversing the phenotype implies structural alterations that are not readily reversible. Pathological hyperplasia of dysfunctional cells is consistent with the paradigm that SMCs play a central role in TAAD pathogenesis, either preventing disease via homeostatic functions or accelerating disease by inappropriate responses.

During aortic development, immature SMCs produce abundant ECM prior to the upregulation of contractile proteins (25). Conversely, mature SMCs express abundant contractile markers and synthesize relatively low levels of ECM as required for vessel wall maintenance and adaptation to altered mechanical loads. Changes in arterial SMC phenotype characterized by increased ECM production and decreased contractile proteins occurs in atherosclerotic plaques as well as during in vitro culture $(40,41)$ and such contractile-to-synthetic modulation is often referred to as dedifferentiation. Although increased production of type I and III collagen is a distinguishing feature of synthetic SMCs (42), such synthesis need not include all ECM proteins produced during development. Indeed, a synthetic phenotype of SMCs is often described in clinical TAAD specimens based on increased expression of the nonstructural glycoprotein osteopontin, normally a minor component of the media (15-17). Similarly, TAADs can exhibit increased type $\mathrm{V}$ and XI but decreased type I and III collagen production, again in contrast to the classical synthetic SMC phenotype (43). Our mouse model of conditional mTOR hyperactivation documents decreased abundance of transcripts for many ECM molecules that contribute to aortic wall structure, including Col1a1, Col1a2, Col3a1, Col4a1, Col5a1, and Col6a1 but increased expression of other transcripts encoding matrix proteins usually detected at low levels in the media, such as Spp1 (encoding osteopontin) and Col8a1. The latter ECM molecules are preferentially expressed by arterial fibroblasts (44); thus, the activated SMCs could be designated as fibroblast-like. Because these populations of SMCs also express increased lysosome-related molecules they may be also interpreted as macrophage-like. Although SMCs with increased phagocytic properties may be classified as macrophages ("big eaters") based on a functional definition, they differ from myeloid cells. Our preference is to avoid designating pathological SMC functions as like that of other cells because there may be overlap with multiple cell types and this need not imply transdifferentiation into other mature somatic lineages. We favor relating the altered cellular phenotype to dominant structural or functional changes at the tissue level - hence degradative SMCs. Our observations build on the multiple functions described for SMCs in arterial health and disease, including perturbations of endocytosis, phagocytosis, and autophagy $(45,46)$. Importantly, different phenotypes of SMCs can coexist within the media, hence revealing considerable cellular heterogeneity both spatially and temporally that may overlap with functions of other mural cell types.

We found that medial degeneration after Tsc1 disruption associates with increased proteolytic activity and macromolecule clearance by a spectrum of degradative SMCs. We also found MMP2 as a major extracellular protease and multiple lysosomal cathepsins as intracellular proteases; phagocytosis, endocytosis, and lysosomal hydrolysis by SMCs all increased. An association of increased MMP activity with TAAD is well documented, but few reports link lysosomal cathepsins to arterial aneurysms and those involve vessels other than the thoracic aorta and may relate to infiltrating leukocytes $(47,48)$. Increased proteolysis is not necessarily pathological if part of adaptive vessel wall remodeling and increased clearance of extracellular debris may be reparative and promote recycling of macromolecules. Notably, myometrial SMCs rich in lysosomes and containing degraded matrix components and phagocytosed erythrocytes characterize the rapidly involuting uterus postpartum that returns to a preinvolutional state within weeks, indicating both a physiological role for degradative myocytes as well as reversible phenotype switching (49). Nevertheless, these same processes can also lead to medial degeneration of the aorta when dominant. Degradative SMCs with both decreased contractile and synthetic functions is not part of the contractileto-synthetic spectrum associated with occlusive arterial disease and argues for a unique phenotype contributing to TAAD.

Phagocytosis, a characteristic macrophage function, is accomplished to a lesser degree by diverse nonhematopoietic cell types, including SMCs (50). Additionally, SMCs acquire several macro- 
phage markers in response to lipid accumulation (51). Although phenotypic modulation of SMCs into phagocytic cells has been described by some investigators as transdifferentiation $(30,51)$, this process defines conversion of one mature somatic cell type into another. Mac-2, Mac-3, and CD68 were first identified by monoclonal antibodies that distinguish tissue macrophages from precursor monocytes, that is, based on functional maturation and not by hematopoietic lineage (52-54). Yet, these antigens are not specific for macrophages; they are expressed at low levels by a variety of nonhematopoietic cells. Subsequent molecular studies identified Mac-2 as GAL3, a multifunctional protein selectively recruited to damaged lysosomes, Mac-3 as LAMP2, a major lysosomal membrane protein, and CD68, also known as LAMP4, as homologous to lysosomal membrane proteins with a significant fraction localizing to lysosomes (55-57). We find subpopulations of SMCs with chronic mTOR activation expressing LAMP2, GAL3, and, to a lesser extent CD68, but not hematopoietic lineage markers or many markers of specialized immune functions. These findings are consistent with our assays of increased lysosomal activity in activated SMCs but not for transdifferentiation of SMCs into macrophages. We also could distinguish SMCs with increased expression of lysosome-related markers from perivascular myeloid cells in hyperlipidemic control mice without Tsc1 disruption, although we examined all aortic cells and did not select rare intimal populations that may exist below the detection limits of our techniques. An advantage of our models is the use of double-reporter strains that decrease artifacts from SMC-macrophage doublets, phagocytosis of one cell type by another, and autofluorescent extracellular debris. Cells with double- as well as singlereporter constructs may, however, exhibit overlapping fluorescence from transgenic proteins and autofluorescent metabolites or particles. Thus, we excluded double-positive $\left(\mathrm{RFP}^{+} / \mathrm{GFP}^{+}\right)$events that were increased in mice fed a high-fat, high-cholesterol diet and constitute a potential source of artifact (58). It is possible that rare SMC-derived phagocytes containing autofluorescent lipid and a more complete repertoire of macrophage markers reside within this population unsuitable for fluorescence analysis. Distinguishing between phagocytic SMCs and conventional macrophages is important for understanding disease mechanisms, but metaplasia at the tissue level occurs from altered cellular functions regardless of the cell of origin.

Chronic mTOR activation is not expected to result in catabolic cellular processes because physiological mTOR activation from nutrient starvation or growth factor withdrawal leads to phosphorylation and decreased nuclear translocation of TFEB, the master transcriptional regulator of lysosomal biogenesis (59). Persistent starvation, however, reactivates mTORC1 and restores the lysosomal pool (60). Additionally, hyperactivation of mTORC1 from $T s c 1$ or Tsc2 disruption upregulates a subset of lysosomal genes and promotes endocytosis (61). Although TFEB expression is decreased in mTOR-activated SMCs, the related transcription factor MITF is increased. MITF was previously described to be stabilized by Wnt signaling and to drive endolysosomal biogenesis (62). Furthermore, $\beta$-catenin activity is increased in SMC tumors resulting from mutations in TSC1 or TSC2, although this effect is celltype specific, as $\beta$-catenin and MITF are decreased in melanocytes in tuberous sclerosis $(63,64)$. Our findings do not exclude roles for
TFEB and TFE3 as additional regulators of lysosomal biogenesis in SMCs under different conditions and multiple regulatory pathways for a degradative phenotype may exist.

Effective prevention of TAAD by rapamycin after $T s c 1$ disruption demonstrates that mechanisms for medial degeneration in this model depend on mTOR. Rapamycin also successfully prevents aortopathy in other experimental models with diverse mechanisms including diminishing SMC hyperplasia, maintaining SMC contractility, reducing synthetic and proinflammatory SMC phenotypes, decreasing protease production, and suppressing leukocyte recruitment and activation (29, 65-68). Translation to clinical trials should be tempered, however, by known side effects such as impaired wound healing and thus possible adverse effects on adventitial fibrosis and tensile strength (68). Targeting specific degradative functions may have fewer side-effects. Because lysosomal function is essentially a housekeeping activity in all nucleated cells, it is unlikely that broad antagonism of lysosomal activity will be well tolerated as chronic preventative therapy for TAAD. Selective inhibitors of lysosomal enzymes or proton pumps may be effective and should be explored.

\section{Methods}

Mice. C57BL/6J (stock no. 000664), Tsc1 fl/fl (stock no. 005680), $m T / m G$ (stock no. 007676), and $A p o E^{-/-}$(stock no. 002052) mice were purchased from the Jackson Laboratory. Myh11-CreER ${ }^{T 2}$ were a gift from Stefan Offermanns (available from Jackson Laboratory as stock no. 019079) and $F b n 1^{\mathrm{C1039G/+}}$ mice were a gift from Harry C. Dietz (available from Jackson Laboratory as stock no. 012885). Mutant strains were bred to the C57BL/6J background for more than 6 generations. Male mice were euthanized at various ages for analysis (the BAC containing Myh11-CreER ${ }^{T 2}$ inserts on the Y chromosome and female mice do not express the construct).

Animal treatment. Cre-Lox recombination was induced by tamoxifen (Sigma-Aldrich) at $200 \mu \mathrm{g} / \mathrm{d}$ p.o. q.d. for 5 days usually starting at 1.5 weeks of age, while controls were treated with vehicle (corn oil) alone. A subgroup of animals received BrdU (Sigma-Aldrich) at $1 \mathrm{mg}$ s.c. q.o.d. from 3 to 5 or 10 to 12 weeks of age. Certain animals were treated with rapamycin (Calbiochem) at $2 \mathrm{mg} / \mathrm{kg} / \mathrm{d}$ i.p. q.d. or DMSO vehicle alone for variable durations as described, including a final dose 12 hours before euthanasia. Some animals received a highfat (40 kcal\% fat), high-cholesterol (1.25\% w/w), cholic acid-free diet (Research Diets, D12108) from 8 to 24 weeks of age.

In situ and ex vivo examination. After euthanasia, the body cavities were opened widely and in situ images of the thoracic aorta were obtained using an SZX16 dissecting microscope with a camera attachment (Olympus). The unpressurized width (external diameter) of the mid-ascending aorta and proximal descending aorta was measured in triplicate. The unpressurized length of aorta was measured along its outer curvature from the ventricular-aortic junction to the proximal origin of the brachiocephalic artery for the ascending segment and from the distal origin of the subclavian artery to the diaphragmatic hiatus for the descending segment. The aorta was then excised from heart to diaphragm, imaged ex vivo, and weighed. The heart and body were also weighed, body length was measured from snout to anus, and body width was measured at the level of the diaphragm. Aortic dimensions were measured from digital images using a calibrated software tool, whereas body length and width were directly measured with a ruler. 
Ultrasound. Transthoracic B-mode images of the thoracic aorta were obtained in lightly isoflurane anesthetized animals using a Vevo 770 high-frequency ultrasound (VisualSonics). Transverse dimensions of the mid-ascending thoracic aorta were measured in triplicate at end-diastole and end-systole. Aortic distension was calculated as the diameter change during the cardiac cycle.

Blood pressure. The aorta was catheterized via the right carotid artery using a 1.4-F Mikro-Tip pressure catheter (Millar Instruments), and blood pressure was measured in lightly anesthetized mice using a PowerLab system (ADInstruments).

Histomorphometry. Aortas were fixed in $4 \%$ paraformaldehyde (PFA) overnight and embedded in paraffin. Serial 5 - $\mu$ m-thick transverse sections were stained in batches by Yale's Research Histology Laboratory with H\&E, Verhoeff-Van Gieson, Verhoeff without counterstain, Masson's trichrome, Sirius red, Alcian blue/Von Kossa, and Oil Red O using standard techniques and an automated system. Morphometry was performed using ImageJ software (http://rsbweb. nih.gov/ij/) after outlining the internal and external elastic laminae. Lumen area was calculated within the internal elastic lamina (because it closely approximates the adjacent endothelial perimeter). Medial area and mean thickness were calculated between the internal and external elastic laminae. The number of medial cells was calculated by counting hematoxylin-stained nuclei and expressed either as cell density (per $\mathrm{mm}^{2}$ ) or as total number of cells (per cross section) and elastin breaks were counted from Verhoeff-Van Gieson-stained transverse sections. Protein was quantified from histological images using ImageJ for monochromatic stains or a custom color segmentation algorithm for polychromatic stains; relative amount was calculated as percentage area of positive staining in vessel wall compartments on tissue sections and integrated density for nuclear and cytoplasmic cellular staining. Similar techniques were used for human specimens, except intima and adventitia thickness was measured and medial cell density was counted in 3 high-power fields in each of the inner, mid, and outer media, averaged, and total cells were extrapolated from the media area calculated from media thickness, adventitia thickness, and external aorta diameter measurements. En face preparations of $4 \%$ PFA-fixed murine aortas were stained with Oil Red O for 1 hour, rinsed in $60 \%$ isopropanol for 10 seconds, and the extent of Oil Red O staining as percentage aorta surface area was measured using Image J.

Transmission electron microscopy. The ascending aortas of 24-week-old mice were fixed (2\% PFA and $2.5 \%$ glutaraldehyde in 0.1 $\mathrm{M}$ sodium cacodylate buffer at $\mathrm{pH}$ 7.4) for 1 hour at room temperature, and then stored in the same buffer at $4^{\circ} \mathrm{C}$ until processing. Specimens were postfixed in $1 \% \mathrm{OsO}_{4}$ in the same buffer at room temperature for 1 hour. After en bloc staining with $2 \%$ aqueous uranyl acetate for 1 hour, the tissue was dehydrated in a graded series of ethanol up to $100 \%$, followed by propylene oxide, and then embedded in EMbed 812 resin and the sample blocks were polymerized at $60^{\circ} \mathrm{C}$ overnight. Thin sections $(60 \mathrm{~nm})$ were cut with an EM UC7 ultramicrotome (Leica) and poststained with $2 \%$ uranyl acetate and lead citrate. Sample grids were examined in a Tecnai G2 Spirit BioTwin transmission electron microscope (FEI) at $80 \mathrm{kV}$ of accelerating voltage and digital images were recorded with a SIS Morada CCD camera (Olympus) with iTEM imaging software.

Western blotting. Animals were fasted overnight prior to euthanasia for signaling studies, except for tests of rapamycin treatment. The thoracic aorta was rapidly excised by sharp dissection to remove sur- rounding loose adipose tissue and flash frozen. Protein was extracted from homogenized aortas using RIPA lysis buffer containing protease and phosphatase inhibitor cocktail tablets (Roche) and boiled in SDS sample buffer for 5 minutes. Equal amounts of protein per sample were separated by SDS-PAGE, transferred electrophoretically to a PVDF membrane (Bio-Rad Laboratories), and blotted with antibodies (catalog numbers from Cell Signaling Technology, unless otherwise noted) against p-S6K-T389 (catalog 9234), S6K (catalog 2708), p-S6-S235/236 (catalog 4858), S6 (catalog 2217), p-4EBP1-T37/46 (catalog 2855), 4EBP1 (catalog 9452), p-AKT-S473 (catalog 4060), AKT (catalog 9272), SMMHC (catalog ab53219, Abcam), SMA (catalog M0851, Dako), SM22 (catalog ab14106, Abcam), p-MLC2-S19 (catalog 3675), MLC2 (catalog 3672), hamartin (catalog 6935), $\beta$-catenin (catalog 610154, BD Biosciences), MITF (catalog 97800), TFE3 (catalog 14779), TFEB (catalog MABE78, Millipore), LAMP1 (catalog ab24170, Abcam), LAMP2 (catalog 1921-01, SouthernBiotech), HSP90 (catalog SAB4300541, Sigma-Aldrich), and $\beta$-actin (catalog A5316, SigmaAldrich) followed by horseradish peroxidase-conjugated secondary antibodies. Bound antibody was detected with Western Lightning Plus-ECL (PerkinElmer). The blotting membrane was usually cut to allow the detection of multiple proteins of markedly different molecular weights. The appropriate molecular weight of each protein was confirmed at the time of analysis and was not annotated in the images.

$R T-P C R$ and quantitative RT-PCR. Crushed tissue was immersed in RLT lysis buffer (Qiagen) and vigorously vortexed. Total RNA was isolated using an RNeasy Mini Kit and DNase Digestion Set (Qiagen) according to the manufacturer's protocol. Reverse transcription reactions were performed using an iScript cDNA Synthesis Kit (Bio-Rad). PCR was performed using cDNA, PCR Greenmix (Sigma-Aldrich), and primers specific for $T s c 1$ exon17 (forward 5'-AGCAGCATGCACTCAGGAACAG-3'; reverse 5'-TCTGGCTATGCAGTTGGGTCAC-3') and Actb (forward 5'-CCAGTTGGTAACAATGCCATGT-3'; reverse 5'-GGCTGTATTCCCCTCCATCG-3'). The solution was separated by $1.5 \%$ agarose gel. Quantitative RT-PCR was performed using a BioRad CFX94 by mixing equal amount of cDNAs, TaqMan gene master mix, and primers for Eln (Mm00514670_m1), Fbn1 (Mm00514908_ m1), Col3a1 (Mm00802300_m1), Myh11 (Mm00443013_m1), Acta2 (Mm00725412_s1), Myl9 (Mm00725412_s1), and Hprt (Mm03024075_m1) from TaqMan. RNase-free $\mathrm{ddH}_{2} \mathrm{O}$ was used as negative controls for qPCR instead of cDNA samples. All reactions were in a $12.5 \mu \mathrm{L}$ volume, in duplicate. PCR amplification consisted of 10 minutes of an initial denaturation step at $95^{\circ} \mathrm{C}$ followed by 40 cycles of PCR at $95^{\circ} \mathrm{C}$ for 15 seconds, and $60^{\circ} \mathrm{C}$ for 1 minute. We confirmed stable expression of several housekeeping genes.

Fluorescence microscopy. Mouse aortas were fixed overnight in $4 \% \mathrm{PFA}$ at $4^{\circ} \mathrm{C}$ and either embedded in paraffin or cryoprotected in $15 \%$ sucrose for 6-8 hours at $4^{\circ} \mathrm{C}$ and embedded in OCT (Tissue-Tek). Human aorta specimens were immediately embedded in OCT. Tissue blocks were sectioned at $5 \mu \mathrm{m}$. For paraffin sections, the slides were dewaxed in xylene and boiled for 30 minutes in citrate buffer (Vector) for antigen retrieval. Alternatively, frozen sections of human aorta were fixed in acetone for 10 minutes at $-20^{\circ} \mathrm{C}$ prior to immunostaining. Paraffin sections were labeled with antibody against BrdU (cata$\log$ ab6326, Abcam) after denaturing with $1 \mathrm{M} \mathrm{HCl}$ for 15 minutes and TUNEL assay (Sigma-Aldrich) was performed according to the manufacturer's instructions. Frozen sections were labeled with phalloidin (Thermo Fisher Scientific) or an antibody against MMP2 (catalog 
ab37150, Abcam), mouse LAMP2 (catalog 1921-01, SouthernBiotech), GAL3 (catalog CL8942AP, Cedarlane), $\beta$-catenin (catalog 610154, BD Biosciences), MITF (catalog MABE78, Millipore), p-S6 (catalog 4858, Cell Signaling Technology), mouse CD45 (catalog 550539, BD Biosciences), CD11b (catalog 550282, BD Biosciences), CD68 (catalog MCA 1957, Bio-Rad), F4/80 (catalog 14-4801-81, Thermo Fisher Scientific), MOMA-2 (catalog MAB1852, Millipore), TER-119 (catalog 116212, BioLegend), mouse and human SMMHC (catalog ab53219, Abcam), human CD45 (catalog MA5-17687, Thermo Fisher Scientific), and human LAMP2 (catalog ab25631, Abcam) or isotype-matched, irrelevant IgG. Detection of unconjugated primary antibodies was visualized with Alexa Fluor 488-, 568-, or 647-conjugated IgG (Invitrogen). Sections were mounted with ProLong Gold Antifade reagent with DAPI (Thermo Fisher Scientific) and imaged using an Axiovert 200M fluorescence imaging microscope (Zeiss) with Volocity software. In selected sections, GFP signal was augmented with an antibody (cata$\log$ A-21311, Thermo Fisher Scientific) and images were acquired with an SP5 confocal microscope (Leica).

Cellular studies, biomechanical assessment, and RNA-seq. These methods are described in the supplemental materials.

Human specimens. Anterior mid-ascending aorta tissue was obtained from patients undergoing aortic surgery or from organ donors. The specimens were processed by the investigators within the operating room to ensure precise anatomical selection and orientation of the tissues. Histomorphometry was performed in formalin-fixed, paraffinembedded transverse sections, and immunostaining was performed on frozen, OCT-embedded transverse sections.

Statistics. Quantitative data are presented as dot plots of individual values with bars representing the mean and SEM or as a box spanning the interquartile range with a line at the median and whiskers extending to the minimum and maximum values; violin plots are similar to box plots with the addition of a rotated kernel density plot on each side. Repeated observations are presented as points representing the mean with connecting lines and error bars representing SEM. Single numerical values are represented by columns. Comparison of continuous variables between 2 groups was by Student's $t$ test, more than 2 groups by 1-way ANOVA for 1 independent variable, 2-way ANOVA for 2 independent variables, and repeated-measures ANOVA for assays over time, followed by Tukey's or Sidak's multiple-comparisons tests if the null hypothesis was rejected by ANOVA. Comparison of categorical variables between 2 groups was by Fisher's exact test and of survival distribution between 2 groups by log-rank test. Correlations were examined by the Spearman test and linear regression of the data modeled the relationship between variables. Probability values were 2 -tailed and $P<0.05$ was considered to indicate statistical significance. Graph construction and statistical analyses were performed with Prism 8.2.0 (GraphPad Software).

Study approval. Animal research protocols were approved by the Institutional Animal Care and Use Committee of Yale University. Human subjects research protocols with a waiver for consent were approved by the Institutional Review Boards of Yale University and the New England Organ Bank.

\section{Author contributions}

GL and GT designed the study. GL, MW, AWC, LQ, PYC, SY, YJ, $\mathrm{CH}, \mathrm{BJ}, \mathrm{AK}$, and $\mathrm{MRB}$ conducted experiments and acquired data. XL performed electron microscopy. GW and SM acquired RNAseq data. NAC, SG, ZC, JRG, and TWC analyzed RNA-seq data. GL, JDH, and GT analyzed and interpreted data. GL, AG, MS, $\mathrm{JDH}$, and GT wrote and edited the manuscript.

\section{Acknowledgments}

This work was supported by Yale's Department of Surgery (Ohse Award and Glenn Fund to GT), the National Natural Science Foundation of China (81600365 to GL), and the NIH (R01 HL146723 and U01 HL142518 to GT and JDH, and via a subcontract to GT and JDH, P01 HL134605 to Dan Rifkin at NYU).

Address correspondence to: George Tellides, 10 Amistad Street 337B, New Haven, Connecticut 06520, USA. Phone: 203.737.2298; Email: george.tellides@yale.edu.
1. El-Hamamsy I, Yacoub MH. Cellular and molecular mechanisms of thoracic aortic aneurysms. Nat Rev Cardiol. 2009;6(12):771-786.

2. Klima T, et al. The morphology of ascending aortic aneurysms. Hum Pathol. 1983;14(9):810-817.

3. Tang PC, et al. Hyperplastic cellular remodeling of the media in ascending thoracic aortic aneurysms. Circulation. 2005;112(8):1098-1105.

4. Rabkin SW. Accentuating and opposing factors leading to development of thoracic aortic aneurysms not due to genetic or inherited conditions. Front Cardiovasc Med. 2015;2:21.

5. Lyon CA, et al. Aneurysm severity is increased by combined Mmp-7 deletion and $\mathrm{N}$-cadherin mimetic (EC4-Fc) over-expression. Sci Rep. 2017;7(1):17342.

6. Guo DC, et al. Mutations in smooth muscle alpha-actin (ACTA2) lead to thoracic aortic aneurysms and dissections. Nat Genet. 2007;39(12):1488-1493.

7. Pannu H, et al. MYH11 mutations result in a distinct vascular pathology driven by insulin-like growth factor 1 and angiotensin II. Hum Mol Genet. 2007;16(20):2453-2462.
8. Larbre F, Loire R, Guibaud P, Lauras B, Weill B. [Clinical and anatomical case of an aortic aneurysm in the course of Bourneville's tuberous sclerosis]. Arch Fr Pediatr. 1971;28(9):975-984.

9. Shepherd CW, Gomez MR, Lie JT, Crowson CS. Causes of death in patients with tuberous sclerosis. Mayo Clin Proc. 1991;66(8):792-796.

10. Kwiatkowski DJ. Rhebbing up mTOR: new insights on TSC1 and TSC2, and the pathogenesis of tuberous sclerosis. Cancer Biol Ther. 2003;2(5):471-476.

11. Cao J, et al. Thoracic aortic disease in tuberous sclerosis complex: molecular pathogenesis and potential therapies in $\mathrm{Tsc}^{+/-}$mice. Hum Mol Genet. 2010;19(10):1908-1920.

12. Martin KA, et al. Rapamycin promotes vascular smooth muscle cell differentiation through insulin receptor substrate-1/phosphatidylinositol 3-kinase/Akt2 feedback signaling. J Biol Chem. 2007;282(49):36112-36120.

13. Campbell GR, Chamley-Campbell JH. Smooth muscle phenotypic modulation: role in atherogenesis. Med Hypotheses. 1981;7(6):729-735.

14. Shankman LS, et al. KLF4-dependent phenotypic modulation of smooth muscle cells has a key role in atherosclerotic plaque pathogenesis. Nat Med. 2015;21(6):628-637.

15. Lesauskaite V, et al. Smooth muscle cells of the media in the dilatative pathology of ascending thoracic aorta: morphology, immunoreactivity for osteopontin, matrix metalloproteinases, and their inhibitors. Hum Pathol. 2001;32(9):1003-1011.

16. Wang L, Zhang J, Fu W, Guo D, Jiang J, Wang Y. Association of smooth muscle cell phenotypes with extracellular matrix disorders in thoracic aortic dissection. J Vasc Surg. 2012;56(6):16981709.e1.

17. Branchetti E, et al. Oxidative stress modulates vascular smooth muscle cell phenotype via CTGF in thoracic aortic aneurysm. Cardiovasc Res. 2013;100(2):316-324.

18. Meikle L, et al. A mouse model of cardiac rhabdomyoma generated by loss of Tsc1 in ventricular myocytes. Hum Mol Genet. 2005;14(3):429-435.

19. Wirth A, et al. G12-G13-LARG-mediated signaling in vascular smooth muscle is required for salt-induced hypertension. Nat Med. 2008;14(1):64-68. 
20. Muzumdar MD, Tasic B, Miyamichi K, Li L, Luo L. A global double-fluorescent Cre reporter mouse. Genesis. 2007;45(9):593-605.

21. Bellini C, et al. Comparison of 10 murine models reveals a distinct biomechanical phenotype in thoracic aortic aneurysms. J R Soc Interface. 2017;14(130):20161036.

22. Zhang HH, Lipovsky AI, Dibble CC, Sahin M, Manning BD. S6K1 regulates GSK3 under conditions of mTOR-dependent feedback inhibition of Akt. Mol Cell. 2006;24(2):185-197.

23. Davies SP, Reddy H, Caivano M, Cohen P. Specificity and mechanism of action of some commonly used protein kinase inhibitors. Biochem J. 2000;351(pt 1):95-105.

24. Thien A, et al. TSC1 activates TGF- $\beta$-Smad $2 / 3$ signaling in growth arrest and epithelial-tomesenchymal transition. Dev Cell. 2015;32(5):617-630.

25. Wagenseil JE, Mecham RP. Vascular extracellular matrix and arterial mechanics. Physiol Rev. 2009;89(3):957-989.

26. Nelms BL, Labosky PA. Transcriptional control of neural crest development. San Rafael, CA: Morgan and Claypool Life Sciences, 2010.

27. Ilicic $\mathrm{T}$, et al. Classification of low quality cells from single-cell RNA-seq data. Genome Biol. 2016;17:29.

28. Shi F, Sottile J. Caveolin-1-dependent beta1 integrin endocytosis is a critical regulator of fibronectin turnover. J Cell Sci. 2008;121(pt 14):2360-2371.

29. Li W, et al. Tgfbr2 disruption in postnatal smooth muscle impairs aortic wall homeostasis. J Clin Invest. 2014;124(2):755-767.

30. Feil S, et al. Transdifferentiation of vascular smooth muscle cells to macrophage-like cells during atherogenesis. Circ Res. 2014;115(7):662-667.

31. Roccabianca S, Figueroa CA, Tellides G, Humphrey JD. Quantification of regional differences in aortic stiffness in the aging human. JMech Behav Biomed Mater. 2014;29:618-634.

32. MacFarlane EG, et al. Lineage-specific events underlie aortic root aneurysm pathogenesis in Loeys-Dietz syndrome. JClin Invest. 2019;129(2):659-675

33. Schlatmann TJ, Becker AE. Histologic changes in the normal aging aorta: implications for dissecting aortic aneurysm. Am J Cardiol. 1977;39(1):13-20.

34. Ihling $\mathrm{C}$, et al. Cystic medial degeneration of the aorta is associated with p53 accumulation, Bax upregulation, apoptotic cell death, and cell proliferation. Heart. 1999;82(3):286-293.

35. Kirsch EW, Radu NC, Gervais M, Allaire E, Loisance DY. Heterogeneity in the remodeling of aneurysms of the ascending aorta with tricuspid aortic valves. J Thorac Cardiovasc Surg. 2006;132(5):1010-1016.

36. Schmid FX, Bielenberg K, Schneider A, Haussler A, Keyser A, Birnbaum D. Ascending aortic aneurysm associated with bicuspid and tricuspid aortic valve: involvement and clinical relevance of smooth muscle cell apoptosis and expression of cell death-initiating proteins. Eur J Cardiothorac
Surg. 2003;23(4):537-543.

37. Schmid FX, et al. Structural and biomolecular changes in aorta and pulmonary trunk of patients with aortic aneurysm and valve disease: implications for the Ross procedure. Eur J Cardiothorac Surg. 2004;25(5):748-753.

38. Clarke MC, et al. Apoptosis of vascular smooth muscle cells induces features of plaque vulnerability in atherosclerosis. Nat Med. 2006;12(9):1075-1080.

39. Mitchell RN, Jonas RA, Schoen FJ. Pathology of explanted cryopreserved allograft heart valves: comparison with aortic valves from orthotopic heart transplants. J Thorac Cardiovasc Surg. 1998;115(1):118-127.

40. Ross R, Wight TN, Strandness E, Thiele B. Human atherosclerosis. I. Cell constitution and characteristics of advanced lesions of the superficial femoral artery. Am J Pathol. 1984;114(1):79-93.

41. Thyberg J, Nilsson J, Palmberg L, Sjölund M. Adult human arterial smooth muscle cells in primary culture. Modulation from contractile to synthetic phenotype. Cell Tissue Res. 1985;239(1):69-74.

42. Burke JM, Balian G, Ross R, Bornstein P. Synthesis of types I and III procollagen and collagen by monkey aortic smooth muscle cells in vitro. Biochemistry. 1977;16(14):3243-3249.

43. Toumpoulis IK, et al. Differential expression of collagen type V and XI alpha-1 in human ascending thoracic aortic aneurysms. Ann Thorac Surg. 2009;88(2):506-513.

44. Vanlandewijck M, et al. A molecular atlas of cell types and zonation in the brain vasculature. Nature. 2018;554(7693):475-480.

45. Lacolley P, Regnault V, Nicoletti A, Li Z, Michel JB. The vascular smooth muscle cell in arterial pathology: a cell that can take on multiple roles. Cardiovasc Res. 2012;95(2):194-204.

46. Clément M, et al. Vascular smooth muscle cell plasticity and autophagy in dissecting aortic aneurysms. Arterioscler Thromb Vasc Biol. 2019;39(6):1149-1159.

47. Gacko M, Chyczewski L. Activity and localization of cathepsin B, D and $\mathrm{G}$ in aortic aneurysm. Int Surg. 1997;82(4):398-402.

48. Aoki T, Kataoka H, Ishibashi R, Nozaki K, Hashimoto N. Cathepsin B, K, and S are expressed in cerebral aneurysms and promote the progression of cerebral aneurysms. Stroke. 2008;39(9):2603-2610.

49. Brandes D, Anton E. Lysosomes in uterine involution: intracytoplasmic degradation of myofilaments and collagen. J Gerontol. 1969;24(1):55-69.

50. Garfield RE, Chacko S, Blose S. Phagocytosis by muscle cells. Lab Invest. 1975;33(4):418-427.

51. Rong JX, Shapiro M, Trogan E, Fisher EA. Transdifferentiation of mouse aortic smooth muscle cells to a macrophage-like state after cholesterol loading. Proc Natl Acad Sci U S A. 2003;100(23):13531-13536.

52. Ho MK, Springer TA. Mac-2, a novel 32,000 Mr mouse macrophage subpopulation-specific antigen defined by monoclonal antibodies.
JImmunol. 1982;128(3):1221-1228.

53. Ho MK, Springer TA. Tissue distribution, structural characterization, and biosynthesis of Mac-3, a macrophage surface glycoprotein exhibiting molecular weight heterogeneity. JBiol Chem. 1983;258(1):636-642.

54. Micklem K, et al. A human macrophageassociated antigen (CD68) detected by six different monoclonal antibodies. Br J Haematol. 1989;73(1):6-11.

55. Maejima I, et al. Autophagy sequesters damaged lysosomes to control lysosomal biogenesis and kidney injury. EMBO J. 2013;32(17):2336-2347.

56. Chen JW, Murphy TL, Willingham MC, Pastan I, August JT. Identification of two lysosomal membrane glycoproteins. JCell Biol. 1985;101(1):85-95.

57. Holness CL, Simmons DL. Molecular cloning of CD68, a human macrophage marker related to lysosomal glycoproteins. Blood. 1993;81(6):1607-1613.

58. Li F, et al. Autofluorescence contributes to false-positive intracellular Foxp3 staining in macrophages: a lesson learned from flow cytometry. J Immunol Methods. 2012;386(1-2):101-107.

59. Settembre C, et al. A lysosome-to-nucleus signalling mechanism senses and regulates the lysosome via mTOR and TFEB. EMBO J. 2012;31(5):1095-1108.

60. Yu L, et al. Termination of autophagy and reformation of lysosomes regulated by mTOR. Nature. 2010;465(7300):942-946.

61. Peña-Llopis S, et al. Regulation of TFEB and V-ATPases by mTORC1. EMBOJ. 2011;30(16):3242-3258.

62. Ploper D, et al. MITF drives endolysosomal biogenesis and potentiates Wnt signaling in melanoma cells. Proc Natl Acad Sci U S A. 2015;112(5):E420-E429.

63. Mak BC, Kenerson HL, Aicher LD, Barnes EA Yeung RS. Aberrant beta-catenin signaling in tuberous sclerosis. Am J Pathol. 2005;167(1):107-116.

64. Cao J, Tyburczy ME, Moss J, Darling TN, Widlund HR, Kwiatkowski DJ. Tuberous sclerosis complex inactivation disrupts melanogenesis via mTORC1 activation. J Clin Invest. 2017;127(1):349-364.

65. Lawrence DM, Singh RS, Franklin DP, Carey DJ, Elmore JR. Rapamycin suppresses experimental aortic aneurysm growth. J Vasc Surg. 2004;40(2):334-338.

66. Rouer M, et al. Rapamycin limits the growth of established experimental abdominal aortic aneurysms. Eur J Vasc Endovasc Surg. 2014;47(5):493-500.

67. Liu R, et al. ARHGAP18 protects against thoracic aortic aneurysm formation by mitigating the synthetic and proinflammatory smooth muscle cell phenotype. Circ Res. 2017;121(5):512-524.

68. Ferruzzi J, et al. Pharmacologically improved contractility protects against aortic dissection in mice with disrupted transforming growth factor- $\beta$ signaling despite compromised extracellular matrix properties. Arterioscler Thromb Vasc Biol. 2016;36(5):919-927. 